\title{
CHROMOSOME PAIRING, RECOMBINATION NODULES AND CHIASMA FORMATION IN THE BASIDIOMYCETE COPRINUS CINEREUS
}

by

PREBEN B. HOLM ${ }^{1}$, SøREN W. RASMUSSEN ${ }^{1}$, DENISE ZICKLER ${ }^{2}$,

BENJAMIN C. LU ${ }^{3}$ and JEAN SAGE'

'Department of Physiology, Carlsberg Laboratory,

Gamle Carlsberg Vej 10, DK-2500 Copenhagen Valby

2Laboratoire de Génétique, Université de Paris-Sud, Centre D'Orsay,

Bâtiment No. 400, 9 1405, Orsay, France

${ }^{3}$ Department of Botany and Genetics, University of Guelph,

Guelph, Ontario, Canada NIG 2Wl 
Keywords: Meiosis, synaptonemal complex, translocation, interlocking, chromosome breakage, crossing over, interference, disjunction

Meiosis in the basidiomycete Coprinus cinereus was analyzed by three dimensional reconstructions of nuclei covering the period from leptotene to telophase II. Crosses involving three different strains (JR52, PR2301 and E991) were used.

The analysis of 94 completely reconstructed nuclei arranged in a temporal sequence according to the morphology of the synaptonemal complex, the centromeres and the centrosomes permitted the following observations and conclusions: (1) The haploid chromosome number of Coprinus cinereus is 13. (2) Reciprocal translocations have been identified in strains PR2301 and E991. In the former strain, the translocation is between chromosomes 3 and 5 and in the latter between chromosomes 1 and 9. (3) Interlockings and chromosome breaks are present during zygotene but at a lower frequency than in organisms with longer chromosomes. The translocation quadrivalents are more often than normal bivalents involved in interlockings and have more chromosome breaks. (4) Tranformation of a translocation quadrivalent into two heteromorphic bivalents was only observed once in agreement with the contention that the turnover of the synaptonemal complex required for this transformation is prevented in bivalent regions where crossing over has occurred. (5) Correction of interlockings by the sbreakage-reunion « mechanism is complete before mid-late pachytene. (6) The presence of two apparently normal bivalents replacing the translocation quadrivalent in at least one, possibly several, cases suggests that a mretranslocation « has taken place, possibly by a mechanism similar to that responsible for the resolution of interlockings. The implications of this possibility are discussed. (7) During early diplotene the synaptonemal complexes are eliminated from the bivalent arms while synaptonemal complex constituents often remain associated with the centromeres and the chiasmata until late diplotene. (8) Homologous centromere regions remain fused at least until early diakinesis. It is the suggested that this association may serve the same function as chiasmata in maintaining the bivalent configuration up to metaphase I and hence improve the chances of a regular disjunction in bivalents without chiasmata. (9). Recombination nodules are readily identified in the central region of the synaptonemal complex from early zygotene to late diplotene. The total number of nodules expected upon completion of synaptonemal complex formation at late zygotene amounts to 37 and is the same as that observed at early pachytene. The total number of nodules is reduced to 26 before midlate pachytene, a reduction similar to that reported in other organisms. (10) An increasing fraction of the nodules becomes larger and surrounded by chromatin during pachytene - diplotene and by late diplotene, all nodules are replaced by small chromatin condensations - chiasmata. (11) The distribution of nodules among and along the bivalents has been analyzed by comparing the observed distributions and those produced by computer simulation of a random positioning of nodules. The analysis reveals a nearly random distribution at late zygotene while during early pachytene and especially pronounced at mid-late pachytene, the distribution of nodules deviates from a random distribution. The comparison furthermore indicates that the placement of recombination nodules on the bivalents of a nucleus is controlled by nodule/bivalent (bivalent arm) interactions while the interaction between nodules appears to be of less importance.

\section{INTRODUCTION}

Detailed analyses based on three dimensional reconstructions of meiotic nuclei have provided comprehensive information on the individual processes of chromosome pairing, crossing over, chiasma formation and disjunction in diploids, polyploids as well as in organisms heterozygous for chromosomal rearrangements. The orga- nisms employed in these investigations include Bombyx (15, 24, 25, 26, 28), Homo (13, 27), Lilium (12), Triticum (10), Zea (8), Drosophila $(3,4,5)$, Saccharomyces (1), Schizophyllum (2), Neurospora (9) and Sordaria (31, 32) among others. Through these studies a common scheme for the basic events of the meiotic prophase at the ultrastructural level has evolved which has

Abbreviations: $s d=$ standard deviation. 
confirmed and extended the classical concept of the processes comprising meiosis (see 14, 29 for recent reviews).

Most of the organisms on which the electron microscopic analyses have been performed have fairly long chromosomes ranging in total length at pachytene from approximately $200 \mu \mathrm{m}$ in Homo and Bombyx to $3,600 \mu \mathrm{m}$ in Lilium. The analysis of chromosome pairing in the ascomycetes Sordaria macrospora (31) and Sordaria humana (32) does, however, indicate that organisms with short chromosomes follow the same basic scheme with respect to chromosome pairing. The evolution of chiasmata from bivalent regions which at late pachytene contain recombination nodules has so far been described only in Bombyx spermatocytes (15) and it is not yet known whether this represents a general feature of meiosis. Finally, extensive analyses of the distribution of recombination nodules among and along the bivalents of a nucleus have revealed rules of their positioning in Drosophila (3, 4, 5), Homo (27), Bombyx (15), Schizophyllum (2), Neurospora (9) and Sordaria $(32,32)$.

In order to further elucidate the processes involved in chromosome pairing, chiasma formation and the distribution of crossovers among and along bivalents, meiosis in the basidiomycete Coprinus cinereus (formerly C. lagopus) was analyzed by serial sectioning and three dimensional reconstruction of nuclei covering the entire meiotic prophase. The small size of the nuclei together with the shortness of the chromosomes and their low number permitted the reconstruction of a large sample of nuclei.

\section{MATERIALS AND METHODS}

Fruiting bodies obtained from dikaryotic cultures of three crosses were analyzed:

Cross 1: JR52, den + (ATCC 26055) $\times$ PR2301, + me-1, (ATCC 26054)

Cross 2: JR52, den $+($ ATCC 26055) $\times$ $\mathrm{E} 991,++$

Cross 3: PR2301, + me-1 (ATCC 26054) $\times$ $\mathrm{E} 991,++$

Strain JR52 carries the morphological marker den (7) and strain PR2301 me-1 is auxotrophic for methionin (21). The culture procedures, as well as the methods for surgical removal of gills from the fruiting body at different stages of meiosis, were the same as those used previously (18).

Two translocations were identified during the course of this study. Initially the cross JR $52 \times$ PR2301 was found to possess a translocation, in the following referred to as translocation 1. In order to determine which of the two strains that carried the translocation, both strains were crossed to strain E991. In the cross JR52 $\times$ E991, a second translocation (translocation 2) was identified and both translocations were subsequently found in the cross PR2301 $\times$ E991. Hence, it can be concluded that strain PR2301 carries translocation 1 and strain E991 translocation 2.

Fixation was carried out as described by LU $(17,19)$ and the procedures used for serial sectioning and three dimensional reconstruction, as well as the computer techniques used for measuring and plotting the karyotypes, have been described in detail by RASMUSSEN and HOLM (27).

\section{RESULTS}

\subsection{Stages of the meiotic prophase}

The presence of the reciprocal translocations in strains PR2301 and E991 (sections 2 and 3.2) did not appear to affect either the normal progression of meiosis, the ultrastructural characteristics of the remainder of the chromosome complement or the nuclear morphology at the different stages of meiotic prophase.

A total of 94 nuclei, covering the period from leptotene to telophase II, were analyzed by serial sectioning and three dimensional reconstruction. The nuclear ultrastructure at these stages, as well as the earlier pre- and postkaryogamic stages, have been described previously $(16,17,19)$ and the following description is therefore limited to new discoveries as well as to those changes in nuclear and centrosomal ultrastructure which are of relevance in establishing the temporal sequence of the reconstructed nuclei.

Typical leptotene nuclei, with lateral components and with telomeres attached to the nuclear envelope, were readily identified, but due to yet incomplete lateral components the chromosome complement could not be fully reconstructed. Pairing and synaptonemal complex formation are initiated at early zygotene at which stage the 

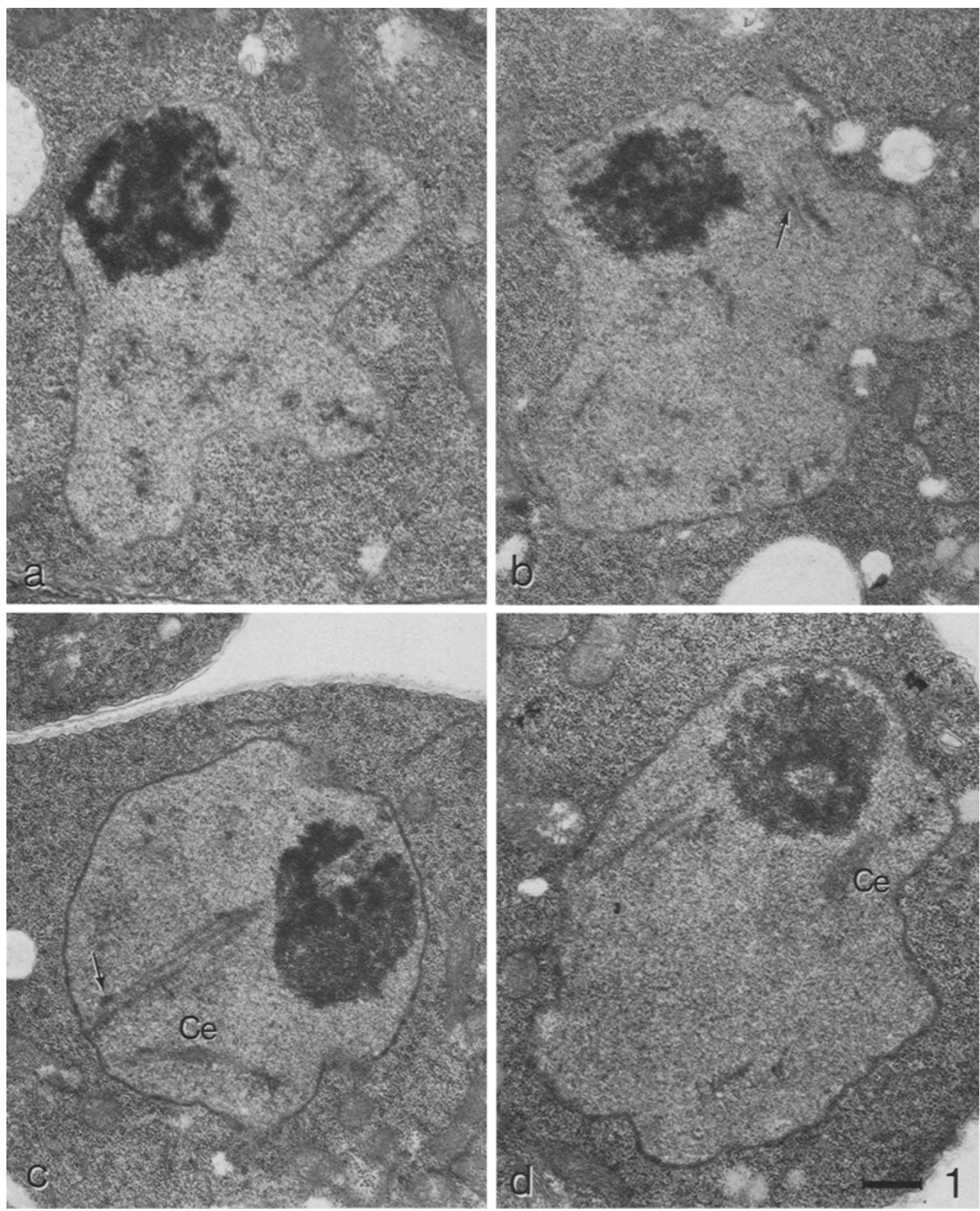

Figure 1. Survey micrographs showing the nuclear morphology at: Late zygotene (Figure 1a); Early pachytene (Figure lb); Mid-late pachytene (Figure lc); Early diplotene (Figure Id); Mid diplotene (Figure le); Late diplotene (Figure 1f); Early diakinesis (Figure 1g); Late diakinesis (Figure $1 \mathrm{~h}$ ); Metaphase I (Figures li and j). The arrows denote recombination nodules (Figures $\mathrm{lb}$ and $\mathrm{c}$ ) or chiasmata (Figure $1 \mathrm{f}$ ). Ce, centromere regions; Ct, centrosome. (Bar $=0.5 \mu \mathrm{m})$ 

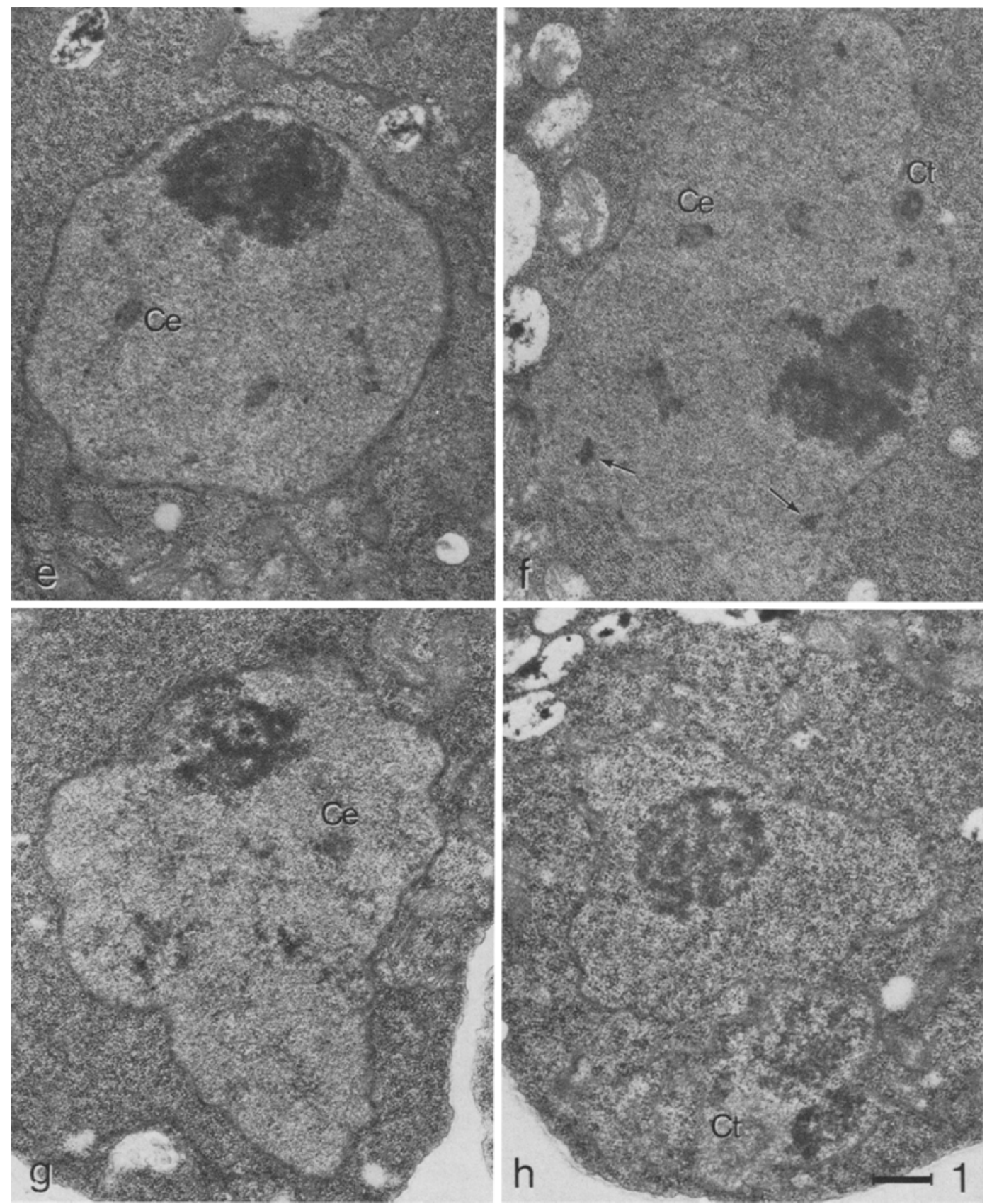

lateral components are continuous from telomere to telomere. Throughout zygotene, the lateral components are distinct with a high electron density, whereas the central region and central component do not yet appear fully organized (Figures $1 \mathrm{a}$ and 17a). The attachment sites of the telomeres are only slightly polarized, and a typical bouquet configuration is not evident. Recombination nodules are frequently present, associated with the central region of the synaptonemal complex (Figures $2 \mathrm{a}$ and 17a. See also section 3.6). A single, electron dense 

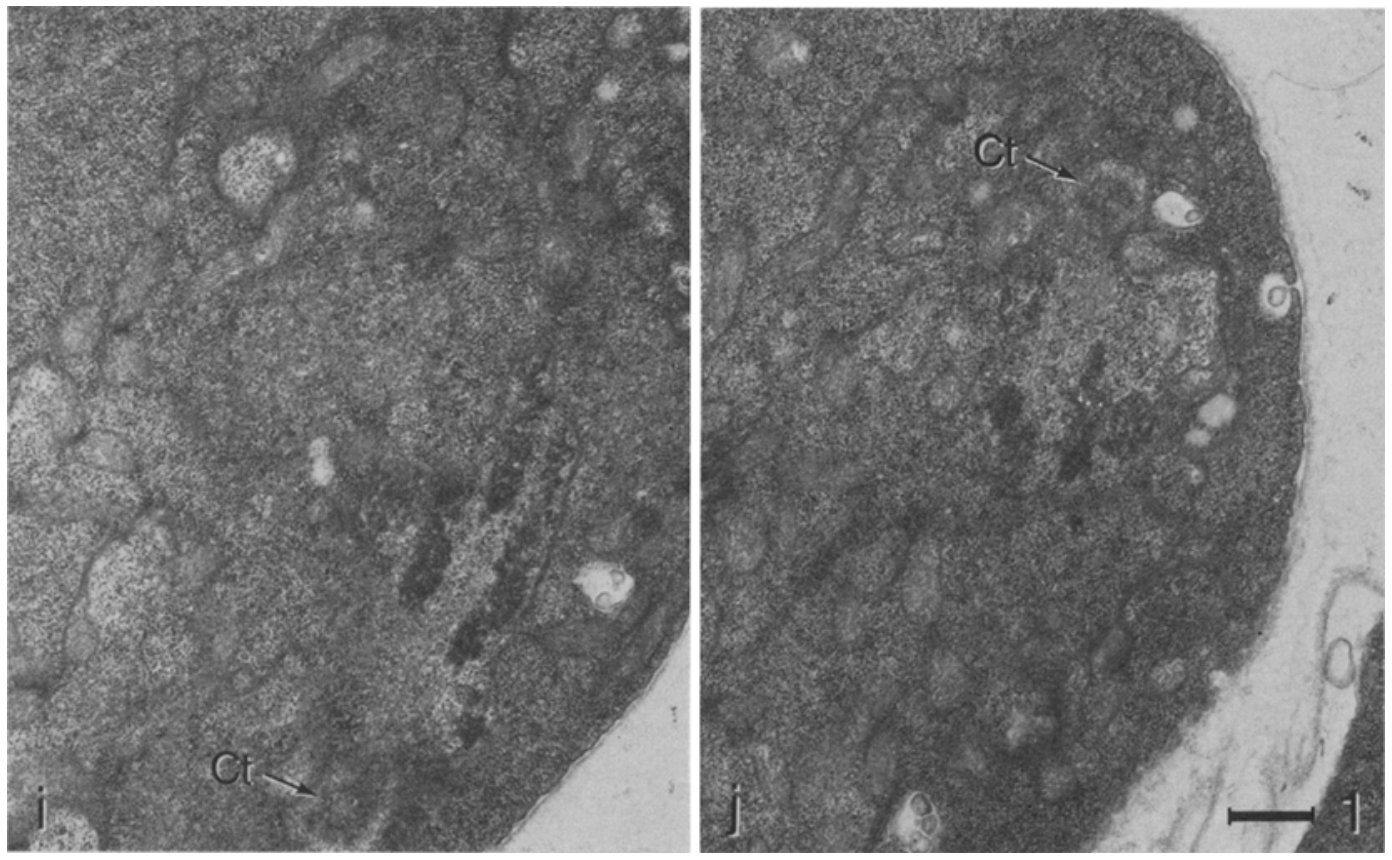

centrosome (spindle pole body) is found in the cytoplasm closely appressed to the outer membrane of the nuclear envelope. The centrosome at zygotene is either spindle or somewhat dumbbell shaped (Figure $3 \mathrm{a}$ ).

Pairing and synaptonemal complex formation are completed by early pachytene (Figure $2 \mathrm{~b}$ ). The lateral components of the complex are still distinct and the central component is more elaborate than at zygotene. Each bivalent possesses one prominent centromere region, consisting of a mass of fibers of medium density, filling most of the central region of the complex. In contrast, centromere regions are hardly recognizable at late zygotene.

Nuclei at mid-late pachytene can be distinguished from early pachytene nuclei by less distinct lateral components and more elaborate central components of the synaptonemal complexes. (Figures $1 \mathrm{c}$ and $17 \mathrm{~b}$ ). Furthermore, the centromere region completely fills and partly surrounds the central region of the synaptonemal complex (Figure $17 \mathrm{~b}$ ). The bivalents, which at late zygotene and early pachytene are often twisted and bended, are at mid-late pachytene more straight and have a lower number of twists (Figure $2 \mathrm{c}$ ).

Synaptonemal complex degradation is initiated at early diplotene (Figures $1 \mathrm{~d}$ and $2 \mathrm{~d}$ ). In most cases, degradation of the lateral components and the central region is initiated in only one of the bivalent arms, more rarely in both arms simultaneously. Reassembly of the constituents of the complex in the form of polycomplexes has not been found. Several of the recombination nodules develop into more open, filamentous and chromatin-like structures which, like the unmodified recombination nodules, are associated with intact synaptonemal complex or with fragments of its central region (Figure 17c). Simultaneously, the chromatin associated with the lateral components disperses, the only condensed chromatin being that associated with centromere regions.

The elimination of synaptonemal complexes from the bivalents continues during mid diplotene, and the individual bivalents can no longer be traced by their synaptonemal complexes (Figures $1 \mathrm{e}$ and $2 \mathrm{e}$ ). The degradation of the complex, the condensation of chromatin around 
the recombination nodules and the condensation of chromatin around the centromeres progress. As shown in Figures $3 \mathrm{~b}$ and c, the centrosomes get more dumb-bell shaped at early diplotene and increase considerably in volume during mid diplotene, as described previously (19). At this and the following stage, the chromosomes are very diffuse and appear as lampbrush chromosomes when analyzed in the light microscope (20).

Synaptonemal complex degradation is virtually complete by late diplotene except for a few remnants in the vicinity of the centromere and all recombination nodules are replaced by condensed chromatin regions - chiasmata - (Fig. ure $1 \mathrm{f})$. The centromeres are generally flanked by two or four regions of compacted chromatin (Figures If and $2 \mathrm{f}$ ), which together with the chiasmata are the only condensed regions present in the nucleus (Figures $1 \mathrm{f}$ and $2 \mathrm{f}$ ). At this stage, as well as at mid diplotene, 13 centromere regions were present in each nucleus.

Condensation of the remainder of the chromatin is initiated at early diakinesis (Figure $1 \mathrm{~g}$ ). In the reconstructed nucleus, only 10 centromere regions were identified, apparently due to close association of two or more centromere regions (Figure $2 \mathrm{~g}$ ). The duplication of the centrosome is completed at early diakinesis, each of the two daughter centrosomes consisting of a sphere of loosely packed electron transparent fibers which in the center contains an electron dense core. Both centrosomes are surrounded by a double membrane which is in contact with the nuclear envelope (Figure $3 \mathrm{~d}$ ).

At late diakinesis, most of the karyoplasm and the nucleolus are expelled from the nucleus (Figures $1 \mathrm{~h}$ and $2 \mathrm{~h}$ ). The bivalents now consist of relatively loosely packed chromatin and more heavily condensed centromeres. Reconstruction of the entire chromosome complement by the contour of the bivalents was, however, not possible due to close association of the individual bivalents. The two centrosomes begin to separate (Figure 4), each one being partly surrounded by a sheet of double membrane. The dense core of the centrosome is at this stage located at the periphery of the less electron dense sphere, facing the bivalents.

At metaphase I, the eliminated karyoplasm, the nucleolus and their surrounding nuclear envelope have nearly disappeared (Figures $1 \mathrm{i}, 1 \mathrm{j}$ and 2 i). The bivalents are heavily compacted. particularly in centromere regions, and numerous microtubules radiate out from the centrosomes. The microtubules are either attached to the centromeres of the bivalents or traverse the metaphase plate and attach to the other centrosome. A nuclear envelope surrounds the bivalents and the centrosomes. The dense core of the centrosomes is less prominent or entirely absent at metaphase I.

\subsection{Translocations}

With a few exceptions, which will be dealt with in section 3.5 , the pairing and synaptonemal complex formation between the translocation chromosomes and their normal homologues gave rise to translocation quadrivalents. The series of micrographs in Figure 5 and the accompanying reconstruction in Figure 6, illustrate the exchange of pairing partners in translocation quadrivalent 1 . Translocation quadrivalent 2 is illustrated by a similar series of micrographs in Figure 7 and a reconstruction in Figure 8. This latter series is from a late zygotene nucleus of the cross PR2301 $\times$ E991 which contains both translocations, Figure 9 showing a reconstruction of translocation quadrivalent 1 from the same nucleus. Generally, both translocations were more readily identified at late zygotene and early pachytene, in particular in the former stage, whereas an unequivocal demonstration of the exchange of pairing partners often was more ambiguous at early and mid-late pachytene as illustrated by the micrographs and the reconstruction of translocation quadrivalent 2 in Figures 10 and 11. The lateral components are at these stages often less distinct in the regions of pairing partner exchange, and the tracing of the chromosomes at this region relies either on weak traces of lateral components or on chromatin connections. A precise determination of the translocation point is therefore often not possible.

As described in detail in section 3.4, translocations 1 and 2 are assumed to involve chromosomes 3 and 5 , and 1 and 9 respectively, the former translocation involving the two long arms and the latter the long arm of chromosome 1 and the short arm of chromosome 9. The reconstruction of the translocation quadrivalents 

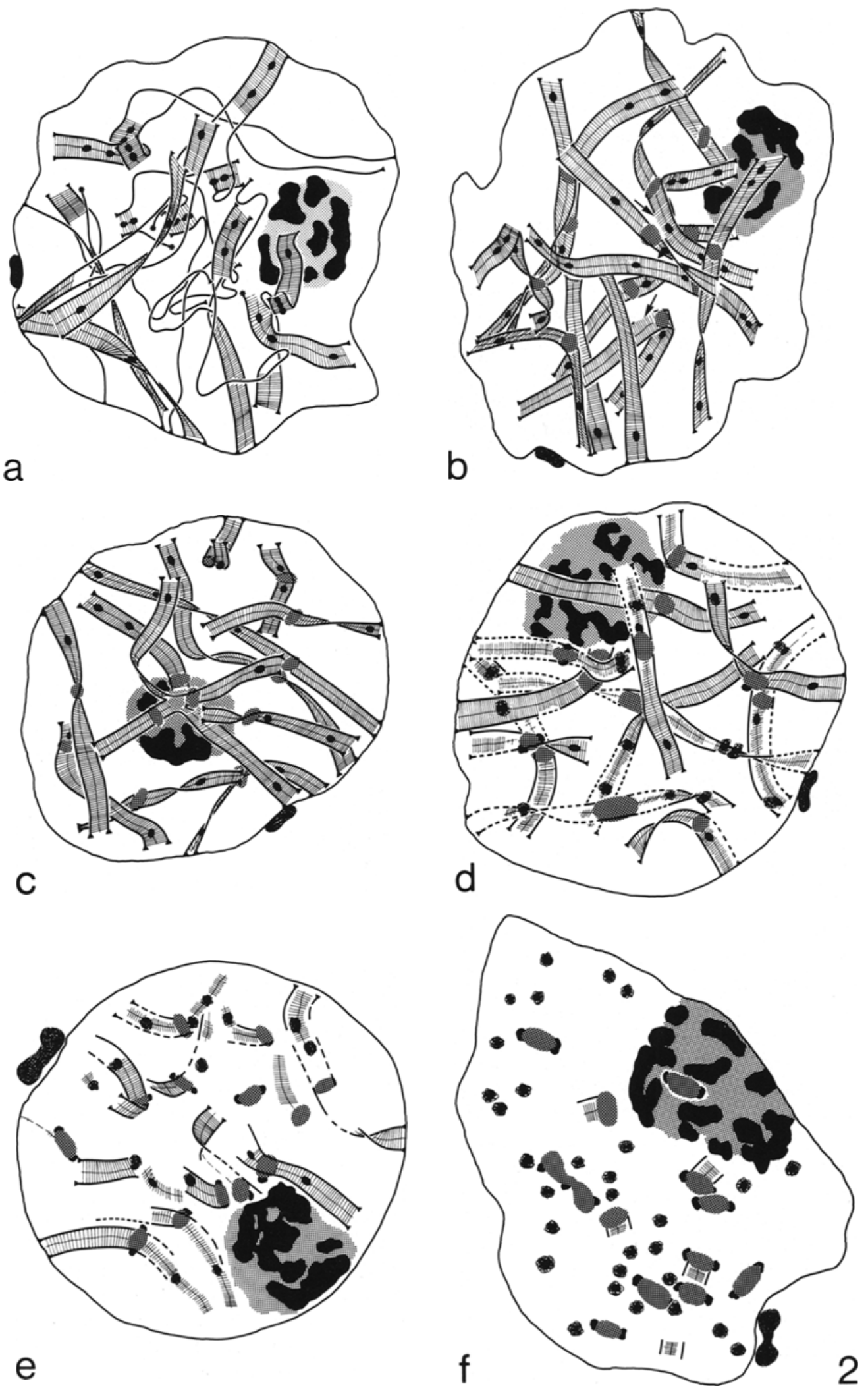

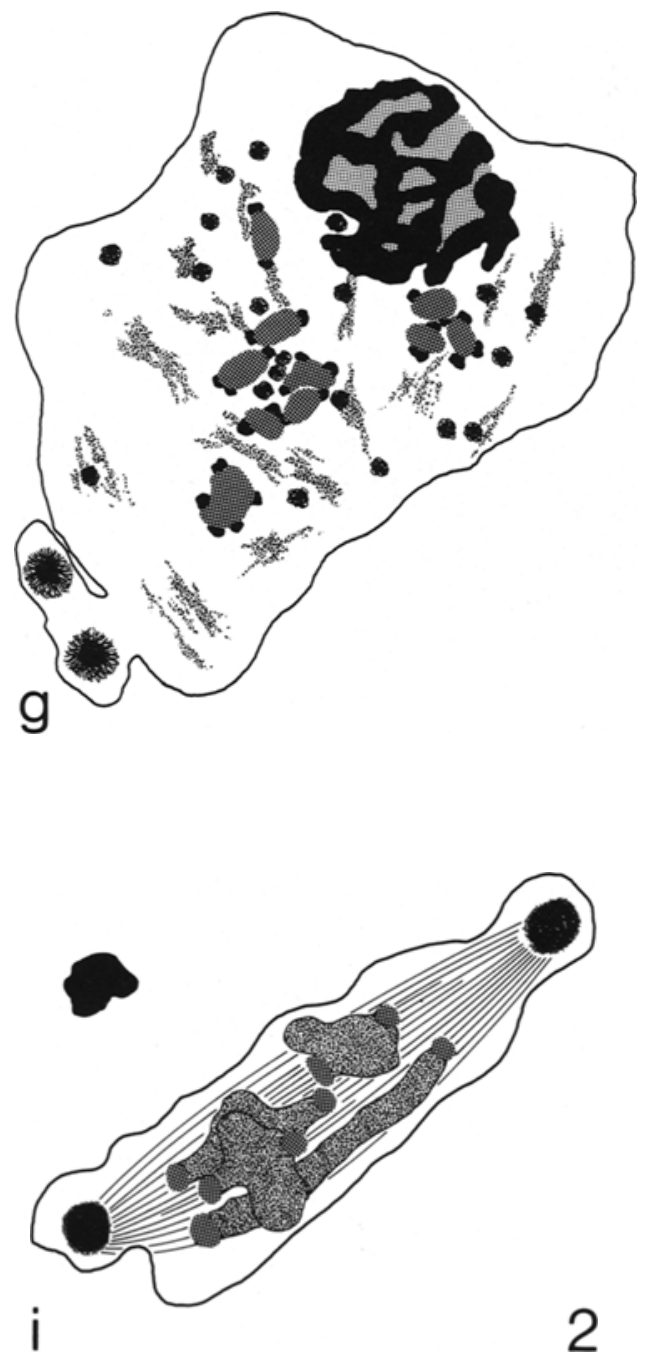

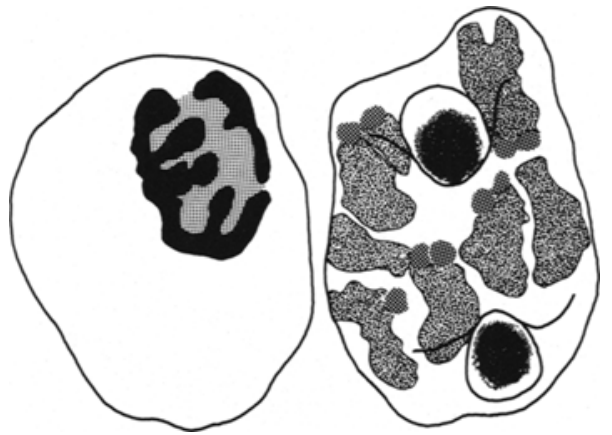

h

Figure 2. Reconstructions of nuclei at: Mid zygotene with translocations 1 and 2 (Figure 2a); Early pachytene, lateral component discontinuities in the chromosomes constituting the translocation quadrivalent 1 are denoted by arrows. (Figure 2b); Mid-late pachytene with translocation 1 (Figure 2c); Early diplotene (Figure 2d); Mid diplotene (Figure 2e); Late diplotene (Figure 2f); Early diakinesis (Figure 2g); Late diakinesis, partial reconstruction (Figure $2 \mathrm{~h}$ ); Metaphase I, partial reconstruction (Figure 2i). From early diplotene onwards, the progressive elimination of the synaptonemal complex from the bivalents prevents the identification of the translocation quadrivalent. (Magnification approximately 15,000.) revealed that the site of pairing partner exchange in both translocation quadrivalents often varied in position along the arm. In order to map the site of the translocation more precisely, the following procedures were used: the translocation chromosomes, i.e., chromosomes 3, 5, 1 and 9 were normalized, the normalization being carried out separately for late zygotene, early pachytene and mid-late pachytene due to the gradual shortening of the bivalents during pachytene (see section 3.4). As described in detail previously (27), the normalization procedure implies an adjustment of each chromosome arm to the mean length of that particular arm with- out changing the relative positions of the pairing partner exchange, and the recombination nodules. The frequencies of pairing in the arms of the translocation quadrivalent were then determined for each $0.2 \mu \mathrm{m}$ interval. As pairing and synaptonemal complex formation are specific at late zygotene and early pachytene (29), the segment where the lowest frequency of pairing was revealed then marks the original breakpoints of the reciprocal translocation. The exchange point for translocation 1 (Figure 12) maps at approximately the same position at late zygotene and early pachytene, whereas the exchange point in bivalent 5 at mid-late pachytene is closer to the 

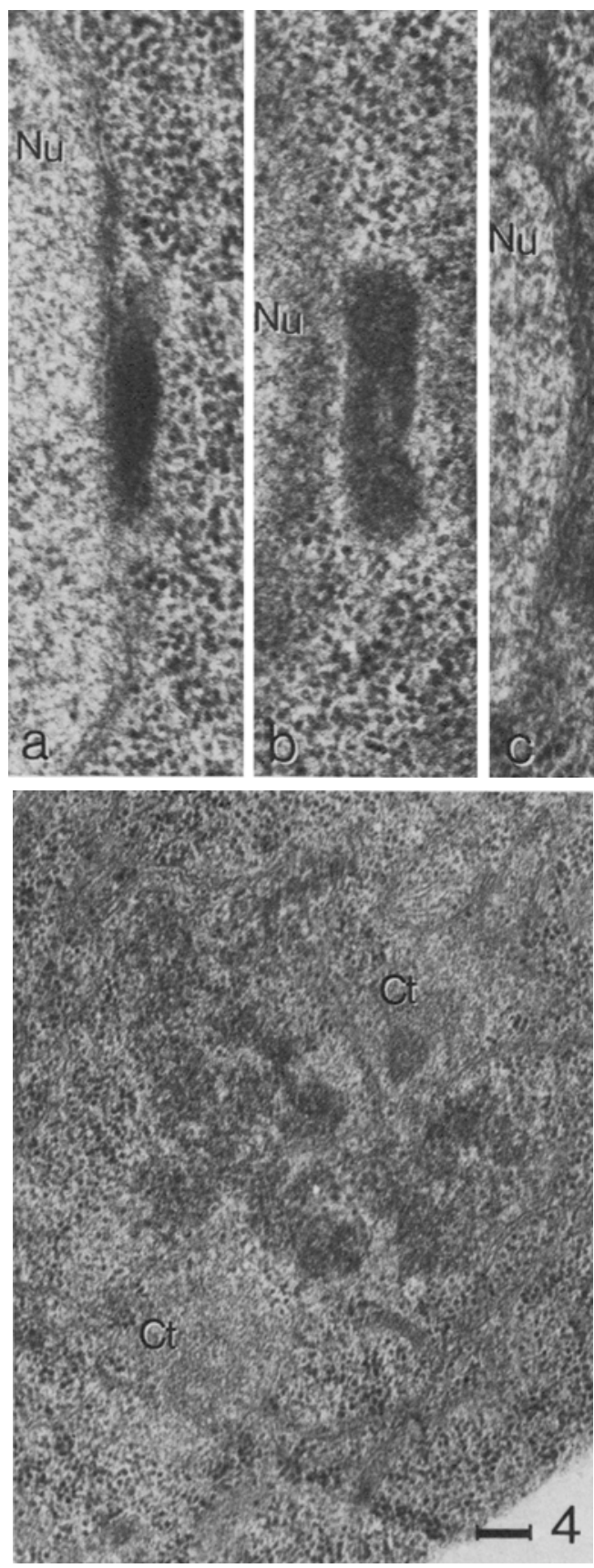

centromere than at the earlier stages. From the late zygotene and early pachytene observations, it can be concluded that the original breakage has taken place at a distance of $0.8 \mu \mathrm{m}$ from the centromere in the long arm of chromosome 3

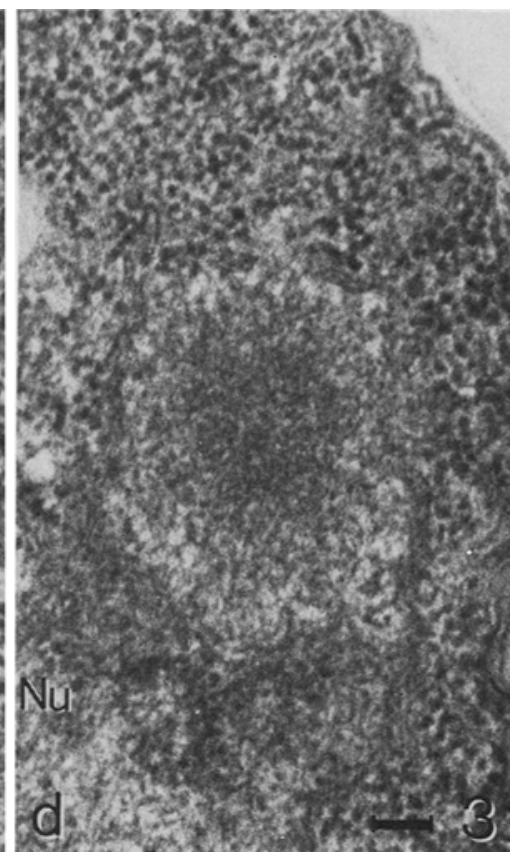

Figures 3 and 4 . Centrosome development from late zygotene to late diakinesis: Late zygotene (Figure 3a); Early diplotene (Figure 3b); Late diplotene (Figure 3c); Early diakinesis (Figure 3d); Late diakinesis, the two centrioles are now completely separated (Figure 4). $\mathrm{Nu}$, nucleus; $\mathrm{Ct}$, centrosome. (Bar: Figure $3=$ $0.1 \mu \mathrm{m}$, Figure $4=0.2 \mu \mathrm{m}$ )

and at a distance of $1.0 \mu \mathrm{m}$ from the centromere in the long arm of chromosome 5, i.e., that the distal $85 \%$ of the long arm of chromosome 3 and the distal $66 \%$ of the long arm of bivalent 5 have been exchanged.

The data for translocation 2 at early and midlate pachytene are shown in Figure 12. At both stages, the exchange point maps at approximately the same position with respect to the centromeres while bivalent 9 is $18 \%$ longer at mid-late pachytene than at early pachytene. Forthermore, the exchange point is in the long arm of bivalent 9 at mid-late pachytene in contrast to the situation at early pachytene.

It is conceivable that the observed increase in length and change in location of the exchange points at mid-late pachytene at least in part are the results of local dissolution of lateral components of the unpaired chromosome regions. 
Table I

Mean values of lateral component (LC) length of one genome, total synaptonemal complex (SC) length, number of recombination nodules ( $\mathrm{RN}$ ) and number of bivalents per nucleus without recombination nodules at mid and late zygotene in Coprinus meiocytes. A, incompletely paired bivalents; B, completely paired bivalents.

\begin{tabular}{cccccccccc}
\hline $\begin{array}{c}\text { Cross } \\
\text { (stage) }\end{array}$ & $\begin{array}{c}\text { Number } \\
\text { of } \\
\text { nuclei }\end{array}$ & $\begin{array}{c}\text { LC length of } \\
\text { one genome } \\
\pm \mathrm{sd}(\mu \mathrm{m})\end{array}$ & $\begin{array}{c}\text { SC length } \\
\pm \mathrm{sd}(\mu \mathrm{m})\end{array}$ & $\begin{array}{c}\text { Percent } \\
\text { pairing }\end{array}$ & $\begin{array}{c}\text { Number } \\
\text { of RN } \\
\pm \mathrm{sd}\end{array}$ & $\begin{array}{c}\text { Expecteda } \\
\text { number of } \\
\mathrm{RN} \pm \mathrm{sd}\end{array}$ & $\begin{array}{c}\text { SC length } \\
\text { per RN } \\
(\mu \mathrm{m})\end{array}$ & $\begin{array}{c}\text { Bivalents } \\
\text { without RN } \\
\mathrm{A}\end{array}$ \\
\hline $\begin{array}{c}\mathrm{JR} \times \mathrm{PR} \\
\text { (mid zygotene) }\end{array}$ & 2 & 48.2 & 26.2 & 54 & 22 & 41 & 1.2 & 0.5 & 0.5 \\
$\begin{array}{c}\mathrm{PR} \times \mathrm{E} \\
\text { (mid zygotene) }\end{array}$ & 1 & 38.7 & 20.1 & 52 & 30 & 58 & 0.7 & 1.0 & 0 \\
\hline $\begin{array}{c}\mathrm{JR} \times \mathrm{PR} \\
\text { (late zygotene) }\end{array}$ & 7 & $42.9 \pm 4.7$ & $36.7 \pm 5.2$ & 86 & $29 \pm 11$ & $34 \pm 13$ & 1.3 & 0.6 & 0.3 \\
$\begin{array}{c}\mathrm{JR} \times \mathrm{E} \\
\text { (late zygotene) }\end{array}$ & 1 & 35.0 & 32.0 & 91 & 24 & 26 & 1.3 & 0 & 0 \\
$\begin{array}{c}\mathrm{PR} \times \mathrm{E} \\
\text { (late zygotene) }\end{array}$ & 2 & 44.4 & 36.1 & 81 & 43 & 53 & 0.8 & 0 & 0 \\
\hline
\end{tabular}

a) The number of recombination nodules expected upon completion of pairing assuming that all regions of the synaptonemal complex will have equal probabilities of receiving a nodule.

\section{Table II}

Mean values of total synaptonemal complex (SC) length and number of recombination nodules (RN) at early and mid-late pachytene in Coprinus meiocytes.

\begin{tabular}{cccccc}
\hline $\begin{array}{c}\text { Cross } \\
\text { (stage) }\end{array}$ & $\begin{array}{c}\text { Number } \\
\text { of nuclei }\end{array}$ & $\begin{array}{c}\text { SC length } \\
\pm \mathrm{sd}(\mu \mathrm{m})\end{array}$ & $\begin{array}{c}\text { Number } \\
\text { of RN } \pm \mathrm{sd}\end{array}$ & $\begin{array}{c}\text { SC length } \\
\text { per RN }(\mu \mathrm{m})\end{array}$ & $\begin{array}{c}\text { Bivalents } \\
\text { without RN }\end{array}$ \\
\hline $\begin{array}{c}\mathrm{JR} \times \mathrm{PR} \\
\text { (early pachytene) }\end{array}$ & 10 & $42.1 \pm 3.0$ & $36 \pm 9$ & 1.2 & 0.4 \\
$\begin{array}{c}\mathrm{JR} \times \mathrm{E} \\
\text { (early pachytene) }\end{array}$ & 3 & $40.2 \pm 1.2$ & $38 \pm 3$ & 1.1 & 0 \\
$\begin{array}{c}\mathrm{PR} \times \mathrm{E} \\
\text { (early pachytene) }\end{array}$ & 5 & $43.4 \pm 2.0$ & $37 \pm 4$ & 1.2 & 0 \\
\hline $\begin{array}{c}\mathrm{JR} \times \mathrm{PR} \\
\text { (mid-late pachytene) }\end{array}$ & 10 & $36.2 \pm 4.6$ & $24 \pm 5$ & 1.5 & 0.3 \\
$\begin{array}{c}\mathrm{JR} \times \mathrm{E} \\
\text { (mid-late pachytene) }\end{array}$ & 11 & $35.8 \pm 2.7$ & $27 \pm 5$ & 1.3 & 0.1 \\
\hline
\end{tabular}

\section{Table III}

Mean values of total synaptonemal complex (SC) length and number of recombination nodules (RN) at mid zygotene, late zygotene, early pachytene and mid-late pachytene in Coprinus meiocytes.

\begin{tabular}{lccccc}
\hline \multicolumn{1}{c}{ Stage } & $\begin{array}{c}\text { Number } \\
\text { of nuclei }\end{array}$ & $\begin{array}{c}\text { SC length } \\
\pm \mathrm{sd}(\mu \mathrm{m})\end{array}$ & $\begin{array}{c}\text { Number } \\
\text { of RN } \pm \mathrm{sd}\end{array}$ & $\begin{array}{c}\text { SC length } \\
\text { per RN }\end{array}$ & $\begin{array}{c}\text { Bivalents } \\
\text { without RN }\end{array}$ \\
\hline Mid zygotene & 3 & $45.0 \pm 7.4 \mathrm{a}$ & $46 \pm 10 \mathrm{~b}$ & 1.0 & 1.0 \\
Late zygotene & 10 & $42.5 \pm 5.0 \mathrm{a}$ & $37 \pm 14 \mathrm{~b}$ & 1.1 & 0.6 \\
Early pachytene & 18 & $42.1 \pm 2.7$ & $37 \pm 8$ & 1.1 & 0.2 \\
Mid-late pachytene & 21 & $36.0 \pm 3.6$ & $26 \pm 5$ & 1.4 & 0.2 \\
\hline
\end{tabular}

a) Total lateral component length divided by two.

b) Number of nodules expected upon completion of pairing (see Table I) 


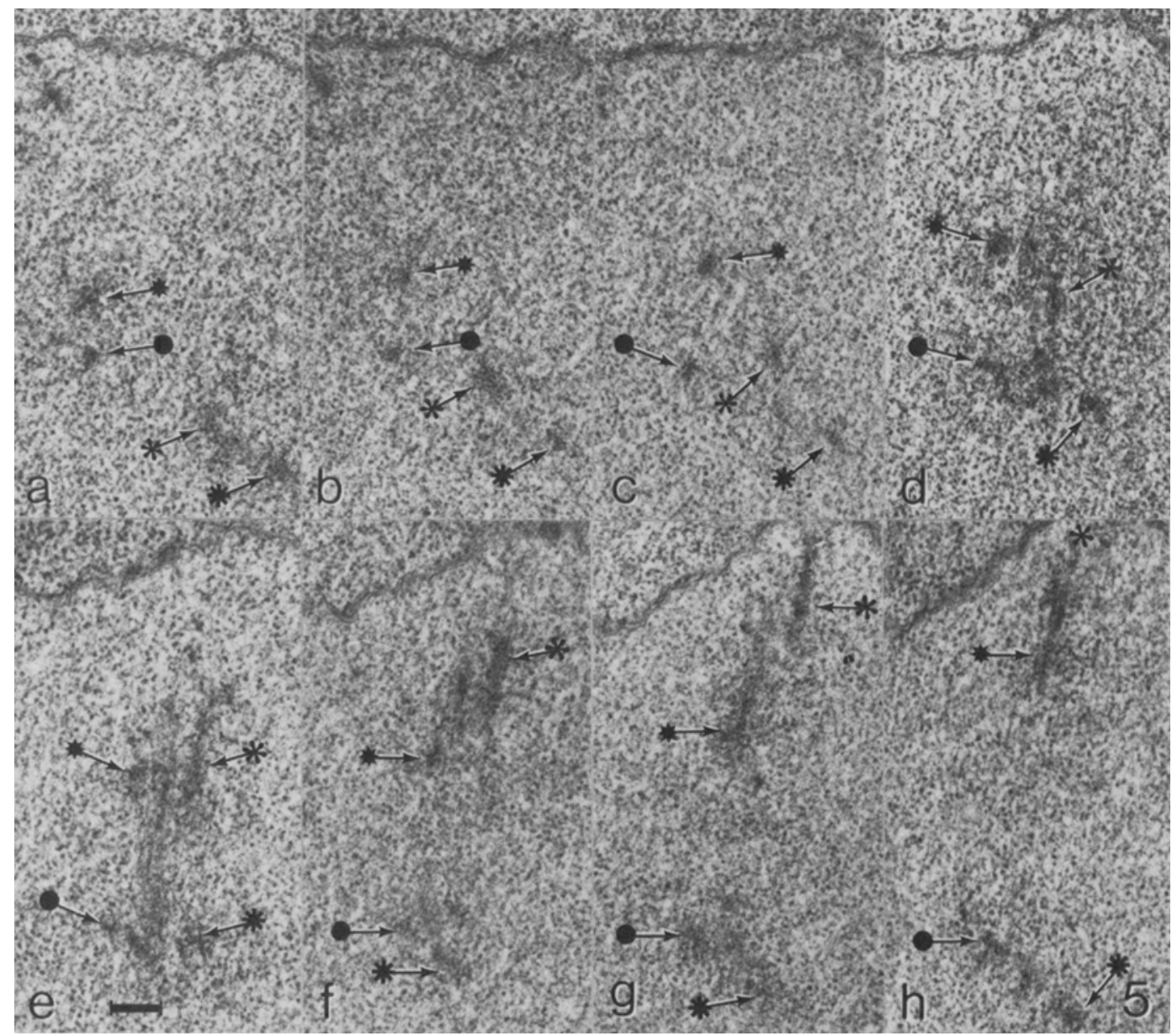

Figures 5 and 6. Eight consecutive sections through the region of pairing partner exchange in translocation quadrivalent 1 at late zygotene.

The nuclear outline and the entire quadrivalent are shown in the reconstruction in Figure 6. The lateral component segments of the exchange region are denoted by arrows in Figure 5 and drawn with thick lines in Figure 6. The letters in Figure 6 refer to the micrographs in which the lateral component segments are present. $($ Bar $=0.2 \mu \mathrm{m})$

On the basis of the early pachytene determination it can be concluded that the initial breakage has occurred $0.9 \mu \mathrm{m}$ from the centromere in the long arm of chromosome 1 and $0.5 \mu \mathrm{m}$ from the centromere in the short arm of chromosome 9, i.e., that $72 \%$ of the long arm of chromosome 1 and $60 \%$ of the short arm of chromosome 9 have been exchanged.

\subsection{Total complement length}

The mean total complement lengths for the three crosses JR52 $\times$ PR2301, JR52 $\times$ E991 and PR2301 $\times$ E991 at mid zygotene, late zygotene, early pachytene and mid-late pachytene are given in Tables I and II. At mid and late zygotene, the mean complement length varies from $35.0 \mu \mathrm{m}$ to $48.2 \mu \mathrm{m}$ and, although the number of observations is considered insufficient for a more detailed comparison, the complement 


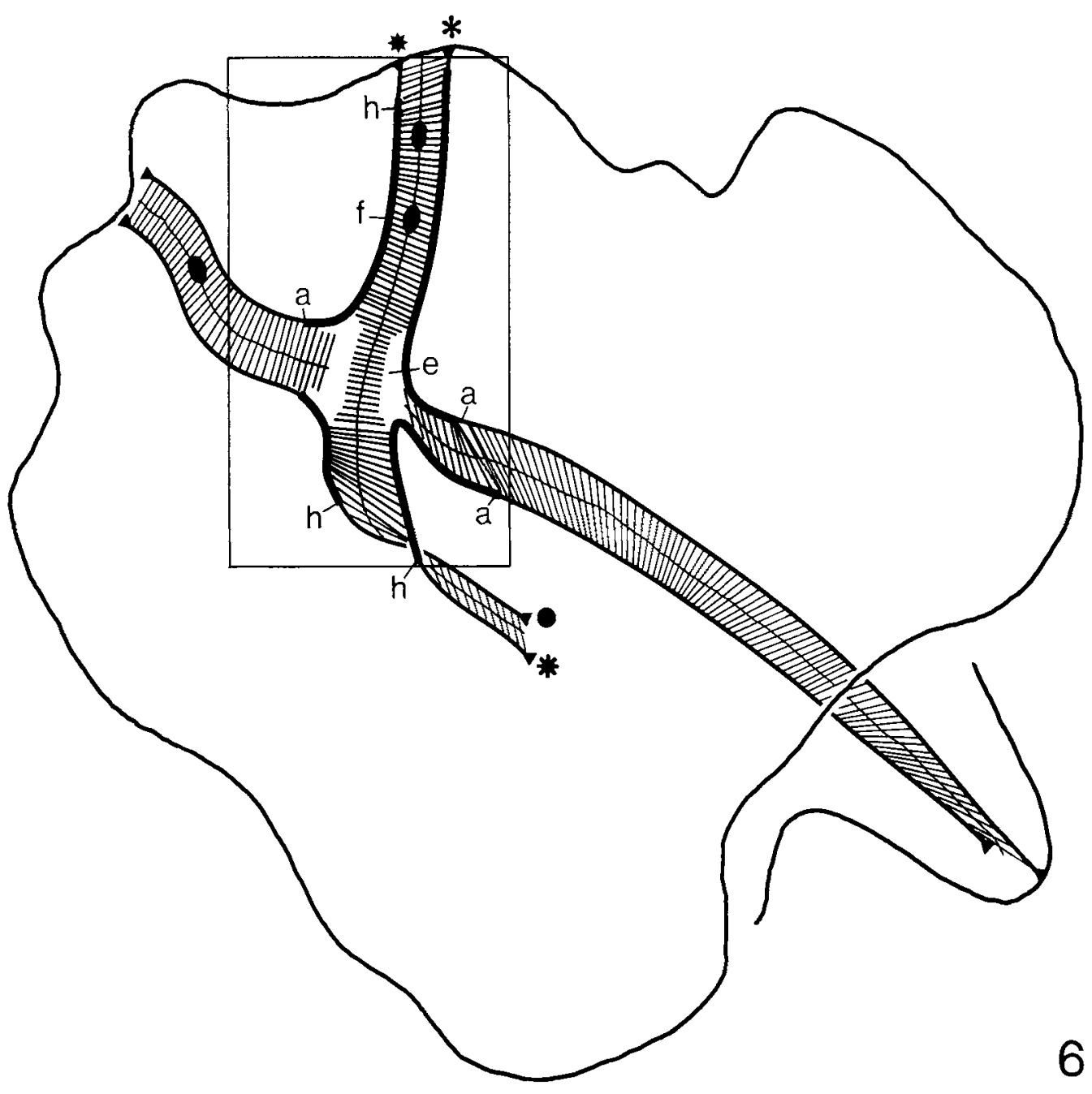

lenghts of the three crosses appear roughly equal. This contention is supported by the early and mid-late pachytene data (Table II) where the more extensive material reveals a similar mean complement length of the three crosses analyzed. At early pachytene the mean values range from $40.2 \mu \mathrm{m}$ to $43.4 \mu \mathrm{m}$ with a standard deviation of only 3 to $7 \%$ of the mean. The agreement between the mean complement length of the two crosses analyzed at mid-late pachytene is even better, with means of $36.2 \mu \mathrm{m}$ and $35.8 \mu \mathrm{m}$. As seen in Table III, the mean complement lengths of all crosses are relatively constant from mid zygotene to early pachytene, whereas the length decreases from $42.1 \mu \mathrm{m}$ to $36.0 \mu \mathrm{m}$ between early and mid-late pachytene. 

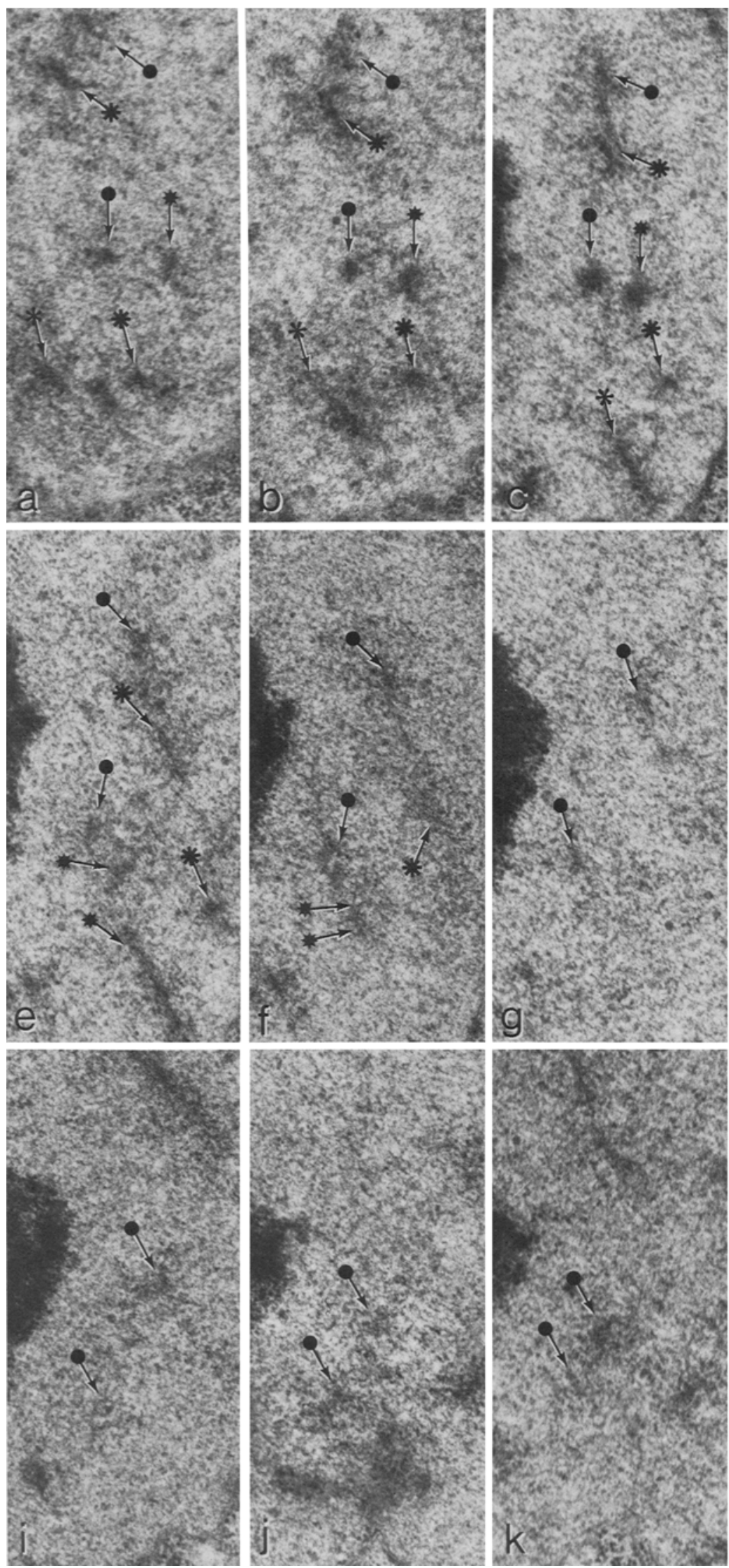
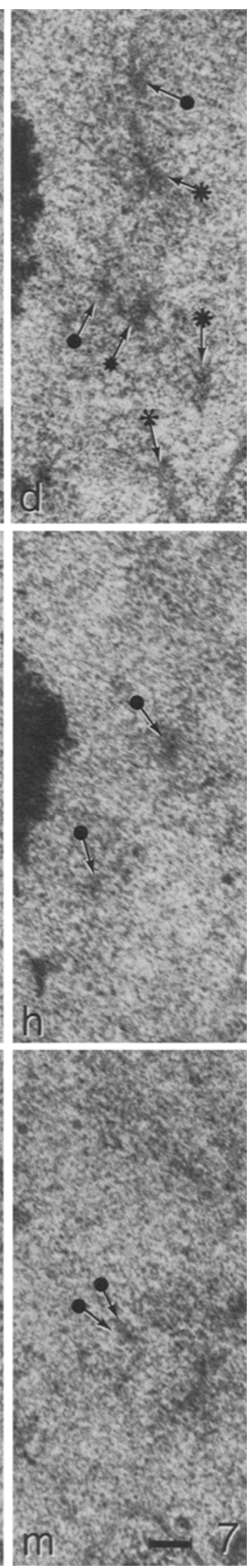


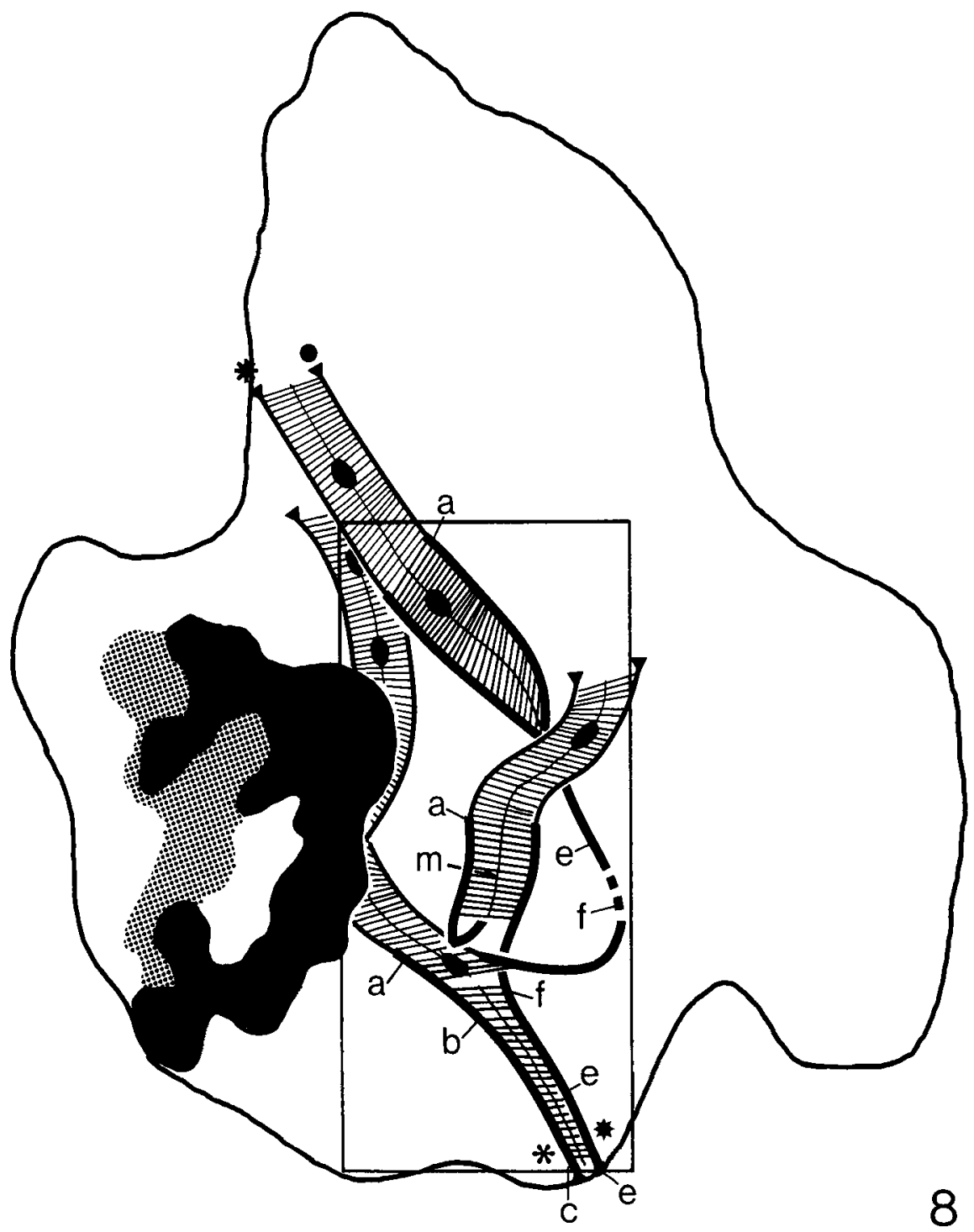

Figures 7 and 8 . Twelve consecutive sections through the region of pairing partner exchange in translocation quadrivalent 2 at late zygotene.

The nuclear outline and the entire quadrivalent are shown in Figure 8. The lateral component segments of the exchange region are denoted by arrows in Figure 7 and drawn with thick lines in Figure 8. The letters in Figure 8 refer to the micrographs in which the lateral component segments are present. $(B a r=0.2 \mu \mathrm{m})$

\subsection{The pachytene karyotype}

The reconstructions have unequivocally revealed that the haploid chromosome number of Coprinus cinereus is 13 in contrast to earlier light microscopic studies of diplotene - diakinesis nuclei which suggested a haploid chromosome number of 12 in cross JR52 $\times$ PR2301
(17). A possible explanation of this discrepancy may be that the translocation quadrivalent was erroneously classified as a single bivalent.

The criteria used in the classification of the individual bivalents were established on the basis of the present material together with the results of an anlysis of the triploid karyotype of $\mathrm{C}$. 


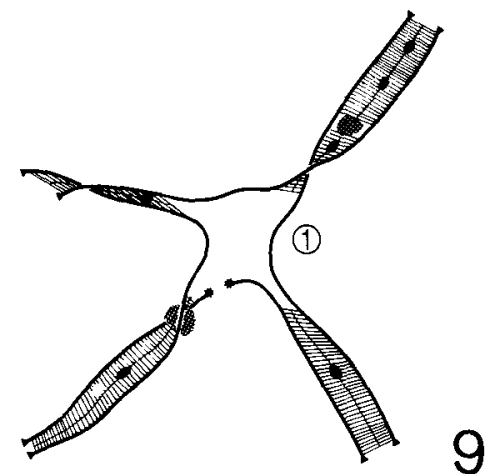

Figure 9. Reconstruction of translocation quadrivalent 1 at late zygotene.

Note the minor discontinuity in one of the chromosomes (denoted by stars) in the region of pairing partner exchange. cinereus (30). The triploid nuclei did not contain translocations and could thus be used as a reference in the identification of the chromosomes involved in the translocations. The following classification is based on absolute length, relative length and centromere index:

Group A: The five longest bivalents arBivalents 1-5 ranged after decreasing length. Bivalent 1 has the lowest centromere index.

Group B: $\quad$ The three bivalents with the lowBivalents 6-8 est centromere index arranged after decreasing length.

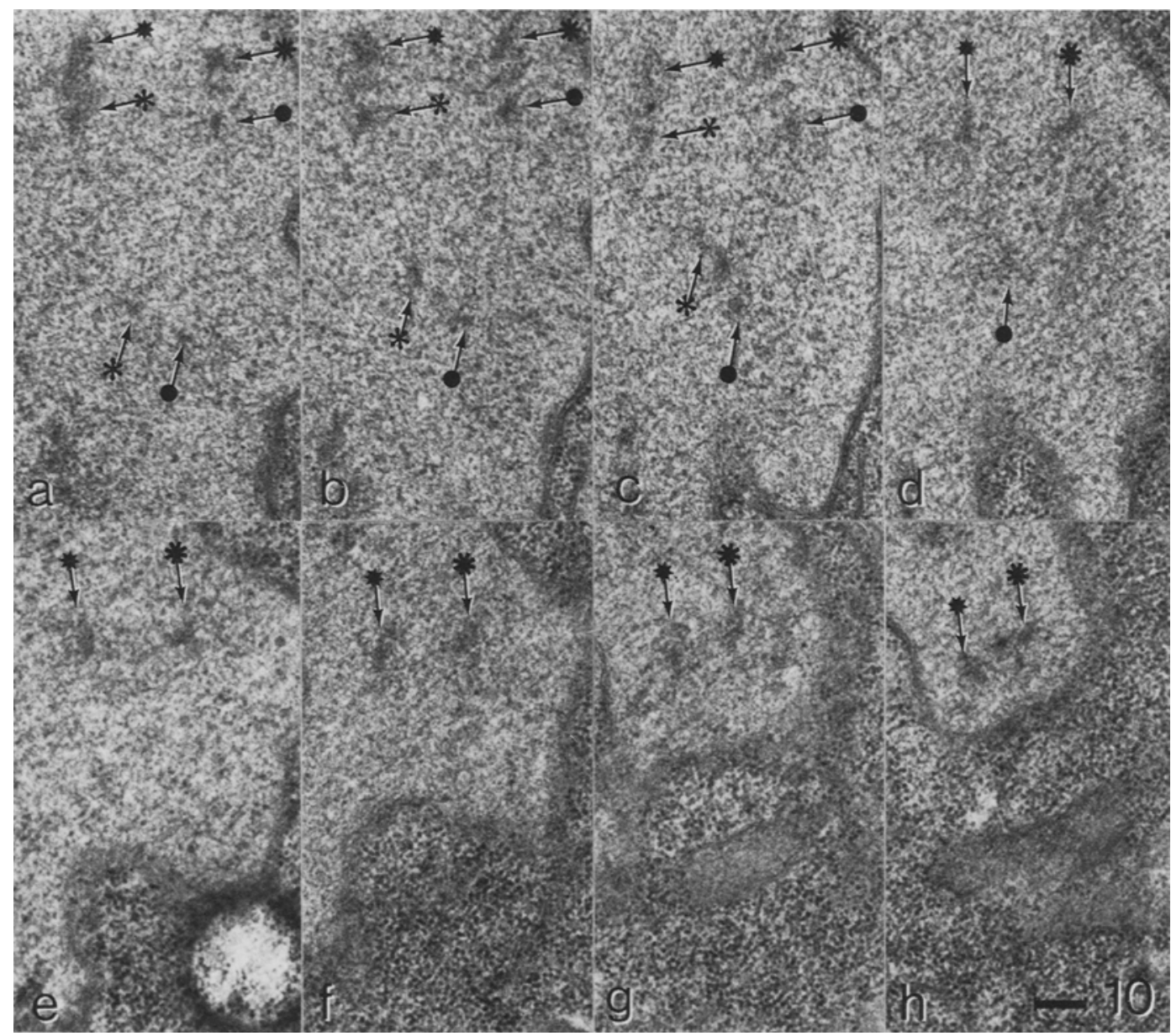

Figures 10 and 11 . Eight consecutive section through the region of pairing partner exchange in translocation quadrivalent 2 at early pachytene.

The continuity of the lateral components is not apparent at the site of the shift in Figure 10, the chromosomes being represented exclusively by their chromatin (as indicated in Figure 11 by the dashed, thick lines in the region designated $\gg d \ll)$. The signatures are the same as in Figures 6 and 8 . (Bar $=0.2 \mu \mathrm{m})$ 
Group C: The four metacentric bivalents Bivalents 9-12 arranged after decreasing length. These bivalents are generally shorter than the bivalents in group B.

Group D: The nucleolus organizing bivaBivalent 13 lent.

A priori, it is not possible to distinguish between translocation and normal, nontranslocation chromosomes in a translocation quadrivalent. Generally, the two chromosome pairs constituting each of the two translocation quadrivalents could be distinguished by differen- ces in length, centromere indices and the position of the exchange of pairing partners (cf. sect. 3.2). The best fit with the triploid karyotype was obtained by designating the shortest and the second longest chromosome of translocation quadrivalent 1 as the nontranslocated chromosomes and the longest and the shortest chromosome as the normal chromosomes of translocation quadrivalent 2. Based on this evaluation and the criteria for the classification presented above. the translocations involve the following chromosomes (Figure 14):

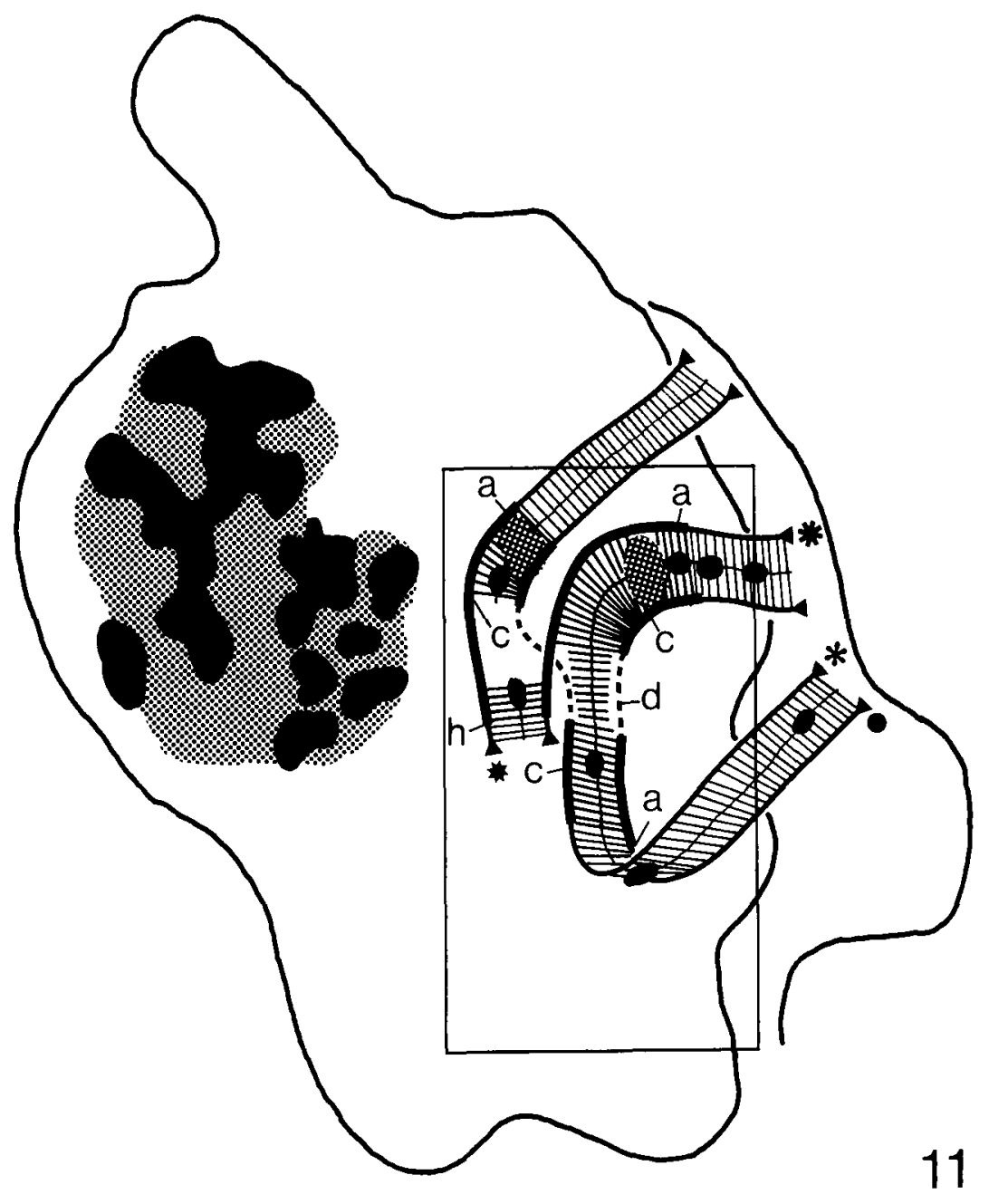


TRANSLOCATION 1

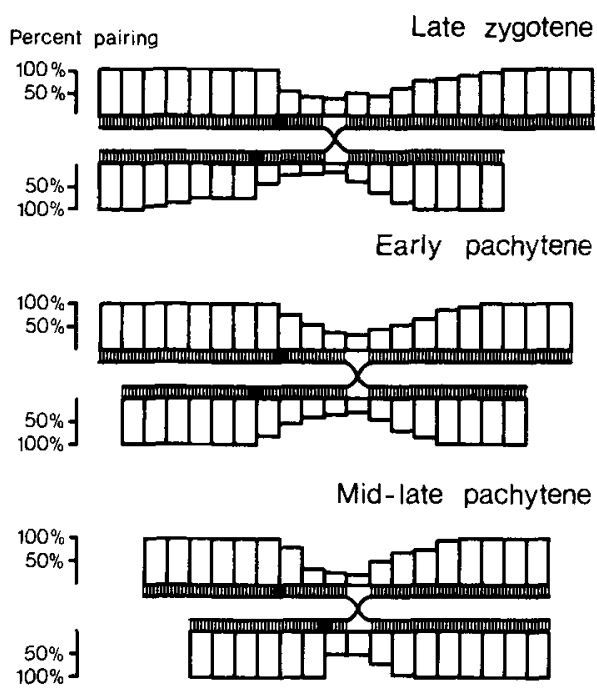

TRANSLOCATION 2

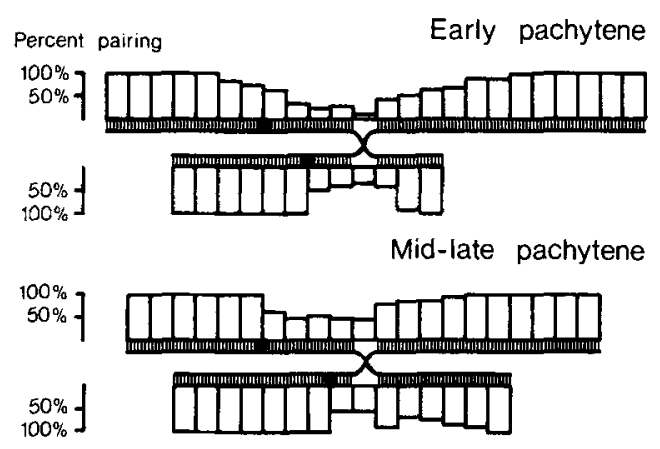

12

Figure 12. Diagrams showing the positions of pairing partner exchanges in the two translocation quadrivalents.

The columns represent the frequencies of completed synaptonemal complex in $0.2 \mu \mathrm{m}$ intervals along the arms. The filled circles mark the position of the centromeres.

Translocation 1: Chromosomes 3 and 5

Translocation 2: Chromosomes 1 and 9

The lengths and centromere indices of the chromosomes involved in the two translocations are given for each of the crosses at early and mid- late pachytene in Table IV. The normal early and mid-late pachytene karyotypes based on lengths and centromere indices for the bivalents and the normal chromosomes of the translocations at early pachytene and mid-late pachytene from each of the crosses are given in Table V. The data from triploid meiocytes are included for comparison and the same results are presented in the idiograms in Figures $13 \mathrm{a}$ and $\mathrm{b}$. Two main conclusions can be drawn from these data: 1) The karyotypes presented from each of the crosses are very similar and do not differ markedly from that of triploid meiocytes. 2) The bivalents can be classified into four groups with reasonable precision. With the exception of bivalents 1 , the longest bivalent, and bivalent 13 , which carries the nucleolus organizing region, the identification is less reliable within the groups and a given bivalent may have received a different number in different nuclei. In Table $\mathrm{V}$ and Figure $13 \mathrm{a}$ and $\mathrm{b}$, the synaptonemal complex length of bivalent 13 is only given for its long arm since in nearly all nuclei the synaptonemal complex of its short arm is either absent or difficult to trace inside the nucleolar material proximal to the centromere region.

\subsection{Chromosome pairing, interlocking and chromosome breakage}

Chromosome pairing and synaptonemal complex formation are generally initiated from the telomeres and only a few examples of interstitial initiation were noted (Figure $15 \mathrm{~d}$ ). In the vast majority of the analyzed nuclei, bivalent formation was regular in the chromosomes not involved in the translocations while pairing and synaptonemal complex formation of the translocation chromosomes and their homologues frequently resulted in irregularities in the form of interlockings and lateral component discontinuities. In all cases, pairing and synaptonemal complex formation were strictly specified and involved only homologous chromosomes and chromosome regions.

All irregularities of chromosome pairing and synaptonemal complex formation revealed at zygotene are summarized in Table VI. The most frequent type is major discontinuities of one or more lateral components in the translocation quadrivalents (Figures $15 \mathrm{a}$ and $\mathrm{c}$ ). In six of the 

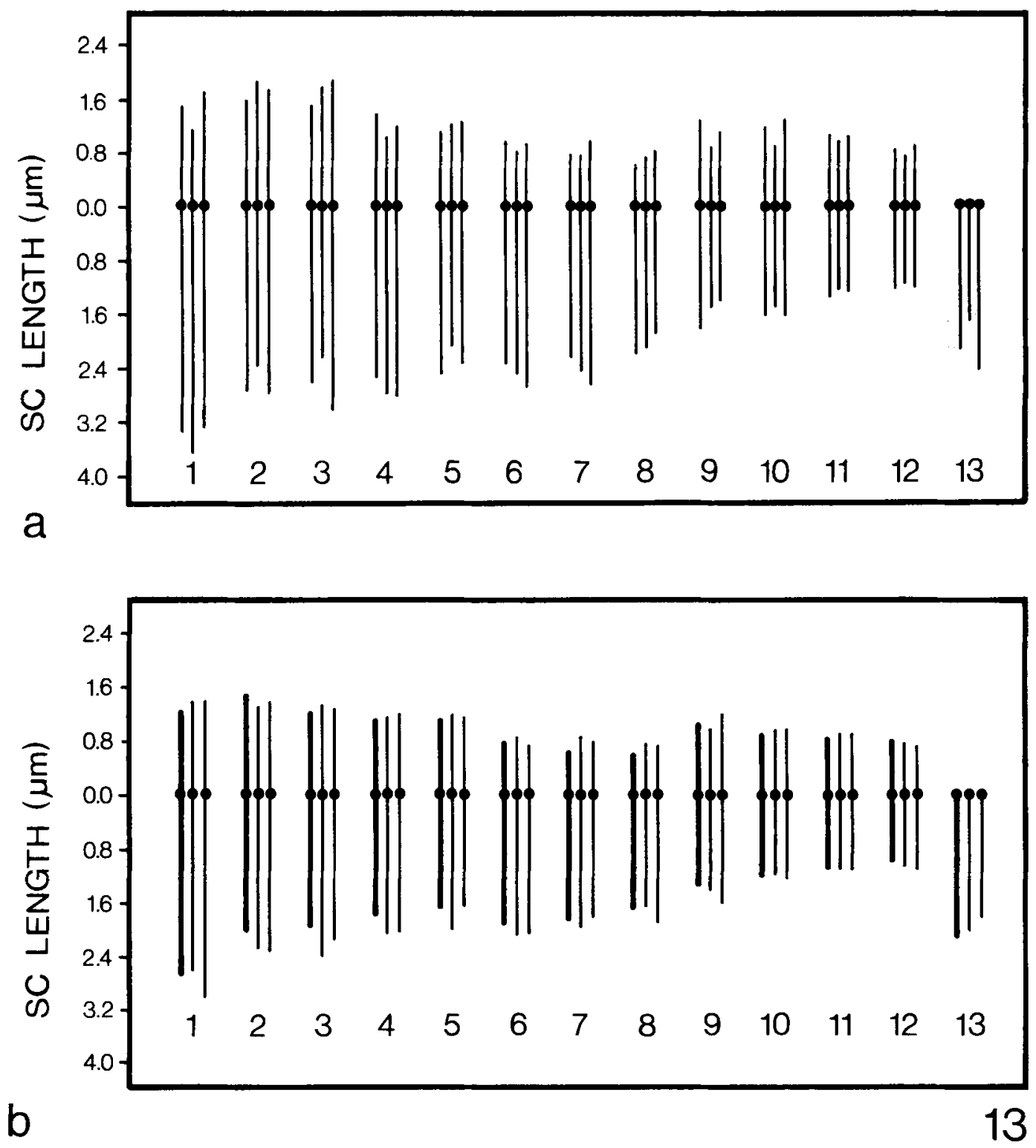

Figure 13. Figure $13 \mathrm{a}$, the early pachytene karyotype of cross JR52 $\times$ PR230I (left lines), cross JR52 $\times$ E99I (middle lines) and cross PR2301 $\times$ E991 (right lines).

Figure 13b, the mid-late pachytene karyotype of triploid Coprinus (left lines), cross JR52 $\times$ PR2301 (middle lines) and cross JR52 $\times$ E991 (right lines).

thirteen zygotene nuclei, one or more of the lateral components of the tranlocation quadrivalent were discontinuous. These discontinuities possibly represent chromosome breakage, as such discontinuities were often found in chromosome regions which appeared to have been interlocked (Figure $15 \mathrm{c}$ ). Breakage of normal chromosomes was found in only 3 nuclei amounting to five chromosome breaks and one bivalent break. Two of the chromosome breaks and the bivalent break had clearly occurred within a region of previous interlocking (Figure 


\section{TRANSLOCATION 1}

Normal chr. 3

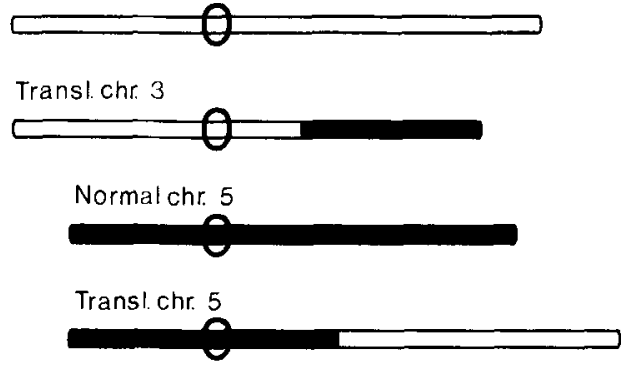

TRANSLOCATION 2

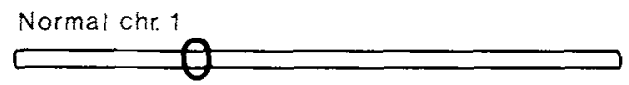

Transl. chr. 1

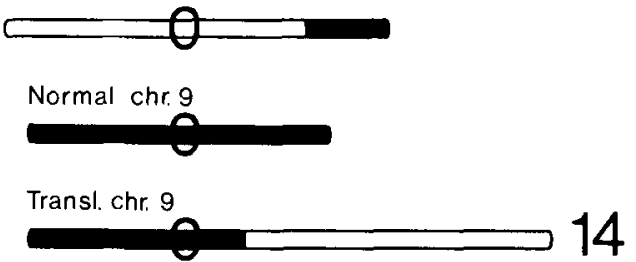

Figure 14. Schematic drawing of the translocation chromosomes and their homologues.
$15 \mathrm{c}$ and d). In addition, the broken bivalent was interlocked by the unpaired lateral components of another bivalent.

Only one example of nonhomologous pairing was observed in the analyzed nuclei: a short synaptonemal complex segment had formed between the two free ends of the broken lateral component of chromosome 9 (Table VI). In summary, the 13 mid-late zygotene nuclei contained one intact bivalent interlocking, four resolving interlockings, one bivalent break and thirteen chromosome breaks, of which seven were in the chromosomes constituting the translocation quadrivalent. When expressed as frequency per nucleus these values corresponds to 0.4 interlockings and 1.2 chromosome breaks per nucleus.

At early pachytene, all irregularities involving the bivalents were resolved and repaired. This was not always the case for the translocation chromosomes and their homologues. One interlocking involving both translocation quadrivalents was found in an early pachytene nucleus from the cross PR2301 $\times$ E991 (Figure 15 b): in this nucleus both the lateral components and

\section{Table IV}

Mean values of absolute length and centromere index of the translocation $(\mathrm{T})$ and non-translocation $(\mathrm{N})$ chromosomes in PR2301 (translocation 1, involving chromosomes 3 and 5) and E991 (translocation 2, involving chromosomes 1 and 9) at early and mid-late pachytene in Coprinus meiocytes.

\begin{tabular}{|c|c|c|c|c|c|c|c|c|}
\hline \multirow{2}{*}{ Translocation 1} & \multicolumn{4}{|c|}{ Absolute length $\pm \mathrm{sd}(\mu \mathrm{m})$} & \multicolumn{4}{|c|}{ Centromere index $\pm \mathrm{sd}(\%)$} \\
\hline & $3 \mathrm{~T}$ & $3 \mathrm{~N}$ & $5 \mathrm{~N}$ & $5 \mathrm{~T}$ & $3 \mathrm{~T}$ & $3 \mathrm{~N}$ & $5 \mathrm{~N}$ & $5 \mathrm{~T}$ \\
\hline $\begin{array}{c}\mathrm{JR} \times \mathrm{PR} \\
\text { (early pachytene) }\end{array}$ & $4.5 \pm 0.5$ & $4.1 \pm 0.3$ & $3.6 \pm 0.4$ & $3.3 \pm 0.5$ & $38 \pm 7$ & $37 \pm 8$ & $31 \pm 10$ & $30 \pm 9$ \\
\hline $\begin{array}{c}\mathrm{PR} \times \mathrm{E} \\
\text { (early pachytene) }\end{array}$ & $5.0 \pm 0.4$ & $4.9 \pm 0.4$ & $3.6 \pm 0.4$ & $3.6 \pm 0.3$ & $34 \pm 7$ & $38 \pm 4$ & $35 \pm 7$ & $37 \pm 9$ \\
\hline $\begin{array}{c}\mathrm{JR} \times \mathrm{PR} \\
\text { (mid-late pachytene) }\end{array}$ & $4.2 \pm 0.3$ & $3.7 \pm 0.4$ & $3.2 \pm 0.4$ & $3.2 \pm 0.4$ & $35 \pm 8$ & $35 \pm 7$ & $37 \pm 7$ & $37 \pm 9$ \\
\hline Translocation 2 & $1 \mathrm{~N}$ & IT & $9 \mathrm{~T}$ & $9 N$ & IN & $1 T$ & 9T & $9 \mathrm{~N}$ \\
\hline $\begin{array}{c}\mathrm{JR} \times \mathrm{E} \\
\text { (early pachytene) }\end{array}$ & $4.9 \pm 0.8$ & $4.8 \pm 0.9$ & $2.6 \pm 0.4$ & $2.4 \pm 0.4$ & $24 \pm 3$ & $29 \pm 7$ & $40 \pm 6$ & $37 \pm 5$ \\
\hline $\begin{array}{c}\mathrm{PR} \times \mathrm{E} \\
\text { (early pachytene) }\end{array}$ & $5.0 \pm 0.4$ & $4.6 \pm 0.5$ & $3.3 \pm 0.6$ & $2.5 \pm 0.5$ & $34 \pm 10$ & $30 \pm 13$ & $42 \pm 8$ & $44 \pm 4$ \\
\hline $\begin{array}{c}\mathrm{JR} \times \mathrm{E} \\
(\text { mid-late pachytene })\end{array}$ & $4.4 \pm 0.6$ & $3.9 \pm 0.5$ & $3.2 \pm 0.5$ & $2.8 \pm 0.5$ & $31 \pm 9$ & $33 \pm 9$ & $41 \pm 8$ & $43 \pm 5$ \\
\hline
\end{tabular}


Table V

Mean absolute length and centromere index of the 13 chromosome pairs in Coprinus meiocytes at early and mid-late pachytene. The mid-late pachytene data from triploid meiocytes ( $3 N$ ) are included for comparison (30).

\begin{tabular}{|c|c|c|c|c|c|c|}
\hline \multirow{2}{*}{$\begin{array}{l}\text { Bivalent } \\
\text { number }\end{array}$} & \multicolumn{3}{|c|}{$\begin{array}{c}\text { Early pachytene } \\
\text { Absolute length } \pm \mathrm{sd}(\mu \mathrm{m})\end{array}$} & \multicolumn{3}{|c|}{$\begin{array}{c}\text { Mid-late pachytene } \\
\text { Absolute length } \pm \mathrm{sd}(\mu \mathrm{m})\end{array}$} \\
\hline & $\mathrm{JR} \times \mathrm{PR}$ & $\mathrm{JR} \times \mathrm{E}$ & $P R \times E$ & $3 \mathrm{~N}$ & $\mathrm{JR} \times \mathrm{PR}$ & $\mathrm{JR} \times \mathrm{E}$ \\
\hline 1 & $4.8 \pm 0.4$ & $4.9 \pm 0.8$ & $5.0 \pm 0.4$ & $3.9 \pm 0.6$ & $4.0 \pm 0.5$ & $4.4 \pm 0.6$ \\
\hline 2 & $4.3 \pm 0.4$ & $4.0 \pm 0.4$ & $4.5 \pm 0.5$ & $3.5 \pm 0.7$ & $3.6 \pm 0.7$ & $3.7 \pm 0.3$ \\
\hline 3 & $4.1 \pm 0.3$ & $4.0 \pm 0.2$ & $4.9 \pm 0.4$ & $3.2 \pm 0.4$ & $3.7 \pm 0.4$ & $3.4 \pm 0.3$ \\
\hline 4 & $3.9 \pm 0.4$ & $3.8 \pm 0.2$ & $4.0 \pm 0.5$ & $2.9 \pm 0.4$ & $3.2 \pm 0.6$ & $3.2 \pm 0.4$ \\
\hline 5 & $3.6 \pm 0.4$ & $3.3 \pm 0.2$ & $3.6 \pm 0.4$ & $2.8 \pm 0.2$ & $3.2 \pm 0.4$ & $2.8 \pm 0.4$ \\
\hline 6 & $3.3 \pm 0.4$ & $3.0 \pm 0.2$ & $3.6 \pm 0.4$ & $2.7 \pm 0.6$ & $2.9 \pm 0.4$ & $2.8 \pm 0.3$ \\
\hline 7 & $3.0 \pm 0.3$ & $3.2 \pm 0.3$ & $3.6 \pm 0.4$ & $2.5 \pm 0.6$ & $2.8 \pm 0.4$ & $2.6 \pm 0.4$ \\
\hline 8 & $2.8 \pm 0.5$ & $2.8 \pm 0.1$ & $2.7 \pm 0.5$ & $2.3 \pm 0.3$ & $2.4 \pm 0.3$ & $2.6 \pm 0.3$ \\
\hline 9 & $3.1 \pm 0.4$ & $2.4 \pm 0.4$ & $2.5 \pm 0.5$ & $2.4 \pm 0.2$ & $2.4 \pm 0.3$ & $2.8 \pm 0.5$ \\
\hline 10 & $2.8 \pm 0.4$ & $2.4 \pm 0.1$ & $2.9 \pm 0.2$ & $2.1 \pm 0.2$ & $2.1 \pm 0.3$ & $2.2 \pm 0.2$ \\
\hline 11 & $2.4 \pm 0.3$ & $2.2 \pm 0.2$ & $2.3 \pm 0.2$ & $1.9 \pm 0.2$ & $2.0 \pm 0.2$ & $2.0 \pm 0.2$ \\
\hline 12 & $2.1 \pm 0.2$ & $1.9 \pm 0.3$ & $2.1 \pm 0.3$ & $1.8 \pm 0.2$ & $1.8 \pm 0.2$ & $1.8 \pm 0.1$ \\
\hline \multirow[t]{2}{*}{13} & $2.1 \pm 0.3$ & $1.7 \pm 0.1$ & $2.4 \pm 0.3$ & $2.1 \pm 0.3$ & $2.0 \pm 0.4$ & $1.8 \pm 0.1$ \\
\hline & \multicolumn{3}{|c|}{ Centromere index } & \multicolumn{3}{|c|}{ Centromere index } \\
\hline 1 & $31 \pm 10$ & $24 \pm 3$ & $34 \pm 10$ & $31 \pm 4$ & $34 \pm 6$ & $31 \pm 9$ \\
\hline 2 & $37 \pm 6$ & $44 \pm 2$ & $38 \pm 7$ & $42 \pm 3$ & $36 \pm 6$ & $37 \pm 5$ \\
\hline 3 & $37 \pm 8$ & $44 \pm 1$ & $38 \pm 4$ & $38 \pm 3$ & $35 \pm 7$ & $37 \pm 6$ \\
\hline 4 & $35 \pm 6$ & $27 \pm 13$ & $29 \pm 10$ & $38 \pm 1$ & $35 \pm 10$ & $37 \pm 9$ \\
\hline 5 & $31 \pm 10$ & $37 \pm 8$ & $35 \pm 7$ & $40 \pm 7$ & $37 \pm 7$ & $41 \pm 4$ \\
\hline 6 & $29 \pm 5$ & $25 \pm 5$ & $25 \pm 1$ & $29 \pm 3$ & $29 \pm 8$ & $26 \pm 9$ \\
\hline 7 & $25 \pm 8$ & $24 \pm 8$ & $27 \pm 8$ & $26 \pm 6$ & $30 \pm 9$ & $30 \pm 9$ \\
\hline 8 & $22 \pm 8$ & $26 \pm 4$ & $30 \pm 11$ & $26 \pm 5$ & $31 \pm 10$ & $28 \pm 10$ \\
\hline 9 & $41 \pm 4$ & $37 \pm 5$ & $44 \pm 4$ & $44 \pm 5$ & $41 \pm 6$ & $43 \pm 5$ \\
\hline 10 & $42 \pm 4$ & $38 \pm 11$ & $44 \pm 5$ & $43 \pm 5$ & $45 \pm 4$ & $45 \pm 4$ \\
\hline 11 & $44 \pm 5$ & $44 \pm 5$ & $45 \pm 3$ & $43 \pm 5$ & $46 \pm 3$ & $46 \pm 2$ \\
\hline 12 & $41 \pm 8$ & $40 \pm 3$ & $43 \pm 5$ & $46 \pm 4$ & $43 \pm 4$ & $41 \pm 5$ \\
\hline 13 & - & - & - & - & - & - \\
\hline
\end{tabular}

the chromatin of two of the chromosomes in translocation quadrivalent 1 were discontinuous over a major distance. In the remainder of the translocation quadrivalents, the lateral components of one or more of the chromosomes were often discontinuous, but as shown in Figures 10 and 11 , the chromatin often appeared continuous and the tracing of the translocations was still possible. Furthermore, the "free« ends of the lateral components were separated by only minor distances, and it is likely that this type of discontinuity does not represent a true chromosome breakage, but rather is the result of a local dissolution of the lateral components leaving the chromatin continuity intact.

In one of the early pachytene nuclei from cross PR2301 × E991, i.e., with both translocations 1 and 2 , translocation 1 could not be identified, the complement consisting of 11 bivalents and translocation quadrivalent 2 . The 

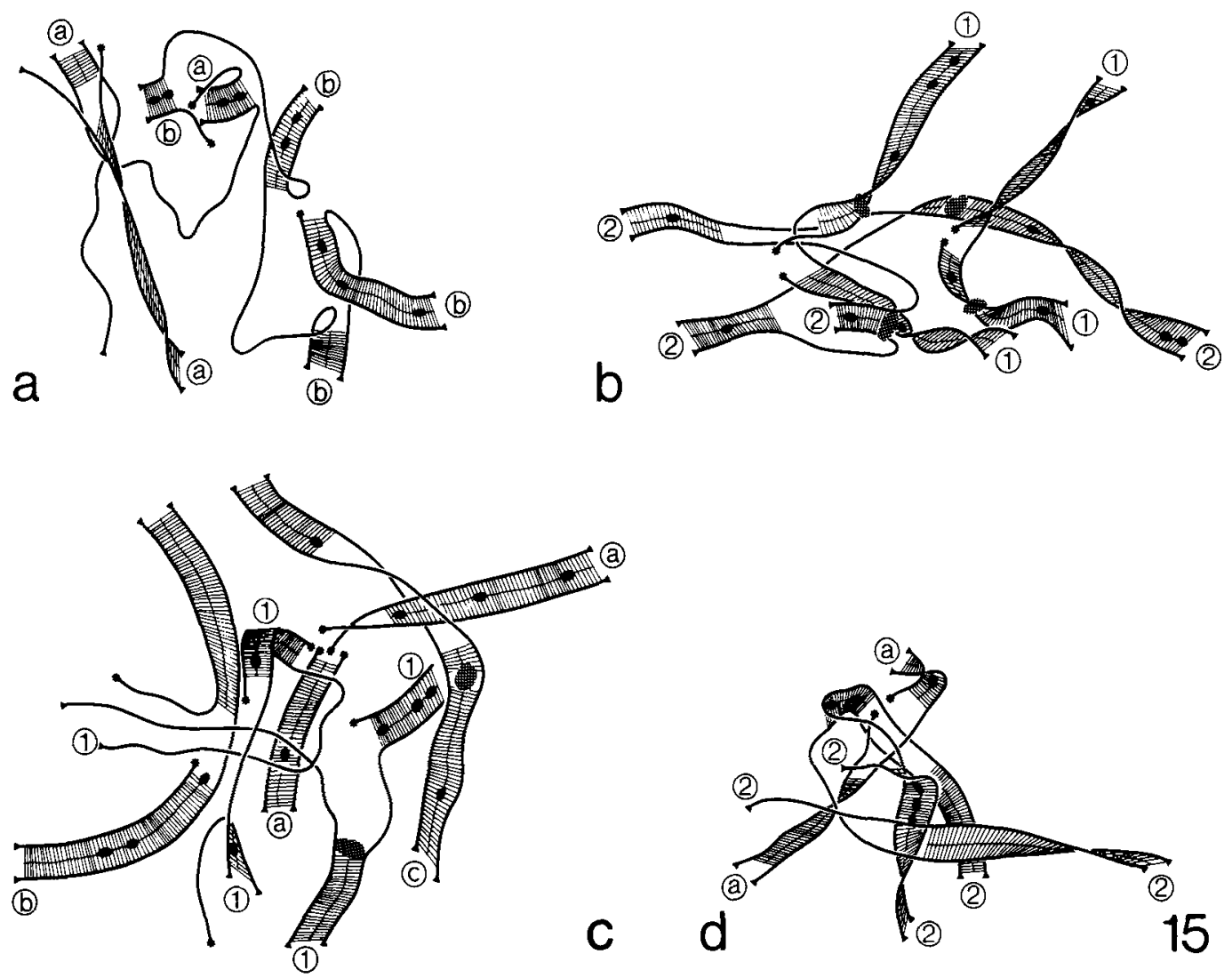

Figure 15. Partial reconstructions of the translocation chromosomes and their homologues.

Figure $15 \mathrm{a}$, the two translocation quadrivalents at mid zygotene. It is not possible to classify the chromosomes constituting the two translocation quadrivalents as the centromeric heterochromatin cannot be distinguished at this stage. Figure $15 \mathrm{~b}$, translocation quadrivalents 1 and 2 at early pachytene. Note that translocation quadrivalent 1 is "resolved « into two bivalents each with a small discontinuity (delimited by stars) in one lateral component. The left bivalent is interlocked by translocation quadrivalent 2 . Figure $15 \mathrm{c}$, complex chromosome configuration involving translocation quadrivalent 1 and three bivalents $(\mathrm{a}, \mathrm{b}$ and $\mathrm{c}$ ). Figure $15 \mathrm{~d}$, resolving interlocking at zygotene involving one chromosome of translocation quadrivalent 2 and a bivalent (denoted a). The free ends of broken lateral components are marked by stars.

lateral components of the synaptonemal complexes were continuous from telomere to telomere in all bivalents including those formed by translocation chromosomes 1 and their normal homologues. This unexpected finding may indicate a wretranslocations « as discussed in section 4.1.

By mid-late pachytene, discontinuities of the unpaired lateral components of the translocation quadrivalents became more pronounced and in some of the 21 investigated nuclei the translocation quadrivalent could not be identified with certainty. Instead, the translocation chromo- somes and their homologues were present as two widely separated bivalents (Figure 16a and Table VI). However, in all cases one lateral component in each of the two bivalents was discontinuous at the presumptive site of pairing partner exchange. In most nuclei two faint chromatin connections were present between the two bivalents while in other nuclei chromatin connections could not be identified. Hence, it is likely that the quadrivalent configuration is maintained in most nuclei but is difficult to resolve in the electron microscope due to discontinuities of the lateral components of the 
Table VI

Descriptions of aberrations in Coprinus meiocytes

\begin{tabular}{|c|c|}
\hline $\begin{array}{l}\text { Nucleus number, } \\
\text { cross }{ }^{\mathrm{a}}\end{array}$ & Aberration \\
\hline \multicolumn{2}{|l|}{ Mid zygotene } \\
\hline $9, \mathrm{JR} \times \mathrm{PR}$ & One of the chromosomes in translocation 1 is broken. \\
\hline 27, JR $\times P R$ & $\begin{array}{l}\text { Two of the chromosomes in the translocation quadrivalent are broken. In the same region } \\
\text { another bivalent is broken, presumably due to the resolution of a previous interlocking. } \\
\text { The broken bivalent is interlocked between the homologues of another bivalent. Two } \\
\text { unpaired chromosome arms in the translocation quadrivalent have previously been } \\
\text { interlocked between another pair of homologues (Figure } 15 \mathrm{c} \text { ). }\end{array}$ \\
\hline $1, \mathrm{PR} \times \mathrm{E}$ & One chromosome in each of the two quadrivalents are broken (Figure $15 \mathrm{a}$ ). \\
\hline \multicolumn{2}{|l|}{ Late zygotene } \\
\hline $21, \mathrm{JR} \times \mathrm{PR}$ & $\begin{array}{l}\text { One homologue of bivalent } 9 \text { is broken and the free ends are nonhomologously paired } \\
\text { with a synaptonemal complex. }\end{array}$ \\
\hline $9, \mathrm{JR} \times \mathrm{E}$ & $\begin{array}{l}\text { One homologue of bivalent } 9 \text { is broken. Normal chromosome } 5 \text { in the translocation } \\
\text { quadrivalent is broken. }\end{array}$ \\
\hline $7, \mathrm{PR} \times \mathrm{E}$ & One chromosome in translocation 1 is broken. \\
\hline $8, \mathrm{PR} \times \mathrm{E}$ & $\begin{array}{l}\text { One bivalent interlocked in translocation quadrivalent. In the same bivalent one of the } \\
\text { chromosomes is broken (Figure } 15 \mathrm{~d} \text { ). One chromosome is missing and one bivalent lacks } \\
\text { a part of one of the homologues. }\end{array}$ \\
\hline
\end{tabular}

Early pachytene

2, $P R \times E$ The two translocation quadrivalents are interlocked. The lateral components of two of the chromosomes in translocation 1 are discontinuous at the presumptive site of pairing partner exchange. (Figure $15 \mathrm{~b}$ ).

4, PR $\times \mathrm{E}$ Translocation 1 cannot be identified because none of the eleven bivalents possess discontinuous lateral components.

Mid-late pachytene

\begin{tabular}{ll}
$7, \mathrm{JR} \times \mathrm{PR}$ & $\begin{array}{l}\text { A translocation quadrivalent cannot be identified. Instead, two bivalents are present each } \\
\text { with one discontinuous lateral component at the presumptive site of pairing partner } \\
24, \mathrm{JR} \times \mathrm{PR}\end{array}$ \\
$\begin{array}{l}\text { exchange. } \\
1, \mathrm{JR} \times \mathrm{E}\end{array}$ & $\begin{array}{l}\text { A translocation quadrivalent cannot be identified. Instead, two bivalents are present each } \\
\text { with one discontinuous lateral component at the presumptive site of pairing partner } \\
\text { exchange (Figure } 16 \mathrm{a} \text { ). }\end{array}$ \\
$7, \mathrm{JR} \times \mathrm{E}$ & $\begin{array}{l}\text { The lateral component of one of the chromosomes in the translocation quadrivalent is } \\
\text { discontinuous over a major distance. }\end{array}$ \\
$14, \mathrm{JR} \times \mathrm{E}$ & $\begin{array}{l}\text { The lateral component of two of the chromosomes in the translocation quadrivalent are } \\
\text { discontinuous over a major distance. }\end{array}$ \\
$16, \mathrm{JR} \times \mathrm{E}$ & $\begin{array}{l}\text { A translocation quadrivalent cannot be identified. Instead, two bivalents are present, one } \\
\text { partly nonhomologously paired, the other with major discontinuities of both lateral } \\
\text { components (Figure } 16 \mathrm{c} \text { ). }\end{array}$ \\
\hline
\end{tabular}

a) Strain PR2301 carries translocation 1 and strain E991 translocation 2.

Carlsberg Res. Commun. Vol, 46, p. 305-346, 198 ! 

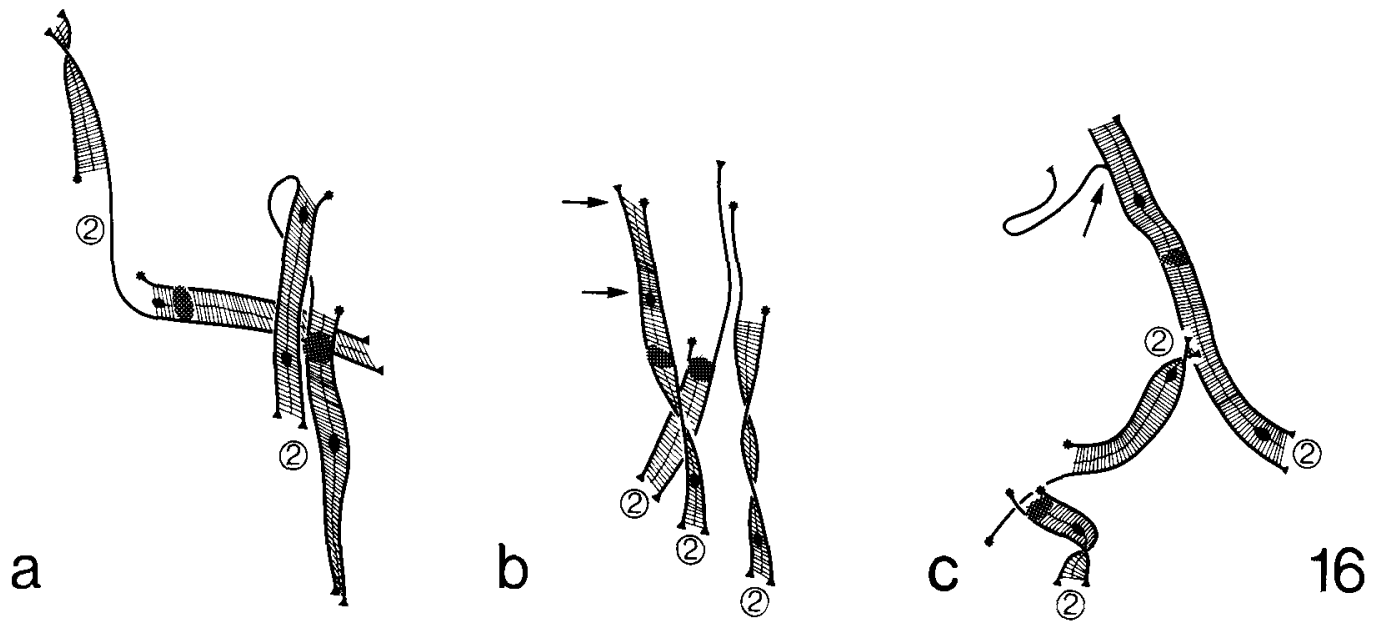

Figure 16. Partial reconstructions of the translocation chromosomes and their homologues at mid-late pachytene.

Figure 16a, apparently "resolved" translocation quadrivalent 2 . Note the major discontinuities (denoted by stars) in one of the lateral components of each bivalent. Figure $16 \mathrm{~b}$, the chromosomes originally constituting translocation quadrivalent 2 . Two chromosome breaks have given rise to three bivalent fragments of which one is nonhomologously paired in one arm (between the arrows). Figure 16c, mresolved « translocation quadrivalent 2. One bivalent is nonhomologously paired over a short distance, the longer chromosome being unpaired at its distal region (the branch point is marked by an arrow). As described in the text, the lateral component segment which completes the synaptonemal complex in the telomeric region of the shorter chromosome is devoid of chromatin. Both lateral components of the other bivalent are broken (denoted by stars).

synaptonemal complex in the region of pairing partner exchange.

An additional case of discontinuity of two lateral components in a translocation quadrivalent is shown in Figure $16 \mathrm{~b}$. A synaptonemal complex is present from telomere to telomere along one of the lateral components, while the other lateral component extends beyond the synaptonemal complex and ends abruptly a short distance from the telomere. In this case, pairing and synaptonemal complex formation are either nonhomologous at the distal region or the lateral component and synaptonemal complex continuity do not reflect the path of the chromosome. The latter possibility is supported by the configuration depicted in Figure $16 \mathrm{c}$. Here, the translocation quadrivalent is resolved into two heteromorphic bivalents. In one of the bivalents, the lateral components are discontinuous at the presumptive site of pairing partner exchange, whereas the other bivalent has an apparently normal synaptonemal complex from telomere to telomere. At a subterminal position in the long arm a long segment of lateral component branches off the complex and is attached to the nuclear envelope at some distance. It is thus apparent that the two chromosomes differ in length and that the synaptonemal complex in the terminal region does not represent a bivalent segment but a segment of an unpaired chromosome where the free face of its central region has associated with a »naked" lateral component giving the structure the appearance of a normal synaptonemal complex.

\subsection{Recombination nodules}

\subsubsection{The transformation of recombination nod- ules into chiasmata.}

Initiation of pairing and synaptonemal complex formation at zygotene is immediately followed by association of recombination nodules to the central region of the synaptonemal complex. These early nodules are more or less spherical, medium electron dense and with diameters of 30-40 nm. As shown in Figure $17 \mathrm{a}$, the nodules are filamentous without a distinct delimitation. In some cases, nodules were attached to partly organized precursor material for the central region of the complex associated 
with one of the lateral components at the pairing fork. During zygotene, a number of structures, to some extent resembling recombination nodules, were present in or associated with the central region. Some of these structures appear as locally disorganized central region material while others resemble electron transparent and partly disorganized recombination nodules. If these structures also represent functional nodules, the number of nodules per nucleus is slightly underestimated (cf. sect. 3.6.2).

At early pachytene, some of the nodules are larger, denser and more elliptical in longitudinal section while the remaining nodules are of the
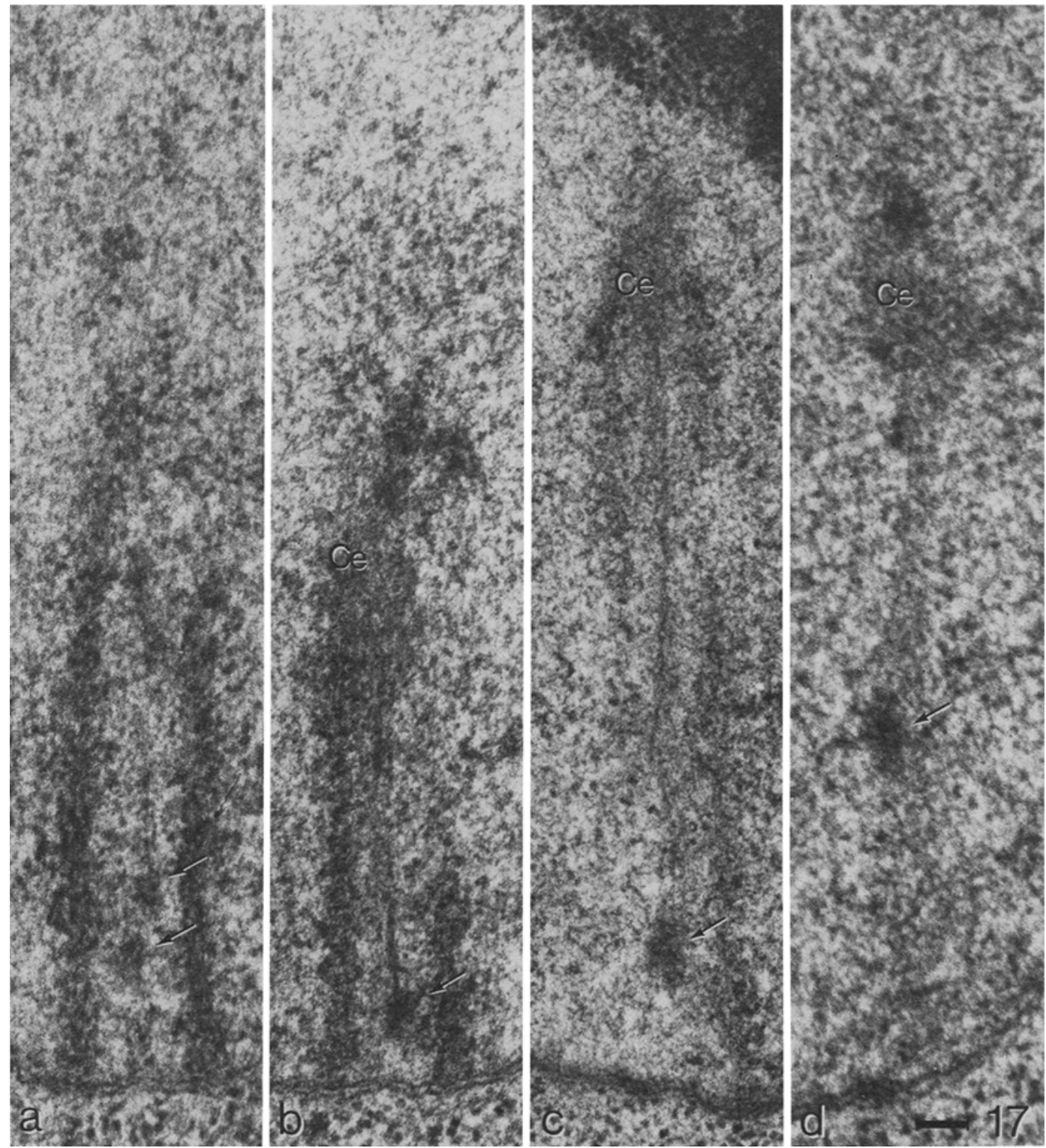

Figure 17. Four longitudinal sections through synaptonemal complexes with recombination nodules (denoted by arrows).

Figure 17a, late zygotene. Figure 17b, mid-late pachytene. Figure 17c, early diplotene. Figure 17d, mid diplotene. $\mathrm{Ce}$, centromere region. $(\mathrm{Bar}=0.1 \mu)$ 


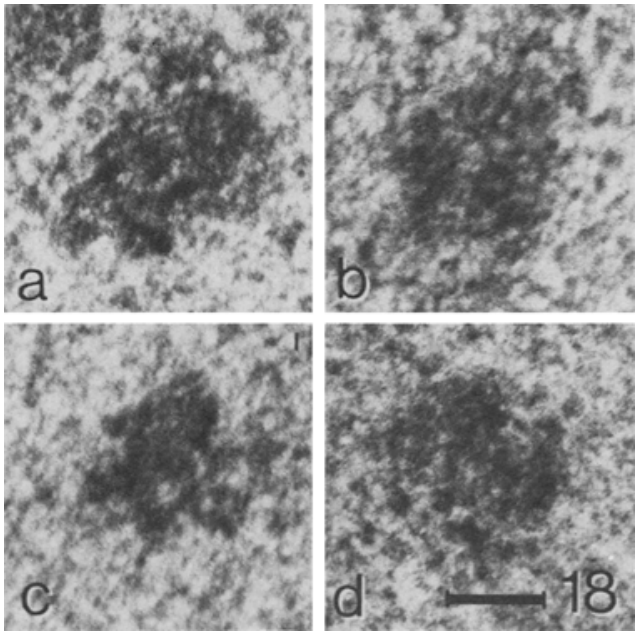

Figure 18. Four micrographs showing the ultrastructure of chiasmata at late diplotene - early diakinesis. $($ Bar $=0.1 \mu \mathrm{m})$

zygotene type. The ratio of these two types of nodules changes during pachytene and at midlate pachytene only the larger, elliptical type is present, always appressed to the central component of the complex. As seen in Figure 17b, this type of nodule consists of an interwoven mass of dense fibers, with a slightly more loose structure than at early pachytene. During late pachyteneearly diplotene, several of the nodules have increased further in size (Figure 17c). At this stage, nodules of the mid-late pachytene type are still present associated with intact segments of synaptonemal complex (Figure 2d). During mid diplotene, the majority of the nodules are large and differentiated into an inner dense core 10-20 $\mathrm{nm}$ in diameter, surrounded by a few dense fibers forming a ringlike structure with a diameter of 50-60 nm (Figure 17d). By late diplotene, nearly all nodules are of this type consisting of a mass of electron dense fibers chiasmata -, which, together with the condensed chromatin flanking the centromeres, represent the only compact chromatin in the nucleus (Figure $2 \mathrm{f}$ ). In several cases, the chiasmata possess a distinct dense core, $10-20 \mathrm{~nm}$ in diameter surrounded by a ring of filaments $60-80 \mathrm{~nm}$ in diameter which, through radiating fibers, are connected to the core (Figures 18a, b and c). In most cases, however, the wheel-like ultrastructure of the chiasmata was less distinct or could not be recognized (Figure $18 \mathrm{c}$ ).

The same organization of the chiasmata is maintained at early diakinesis. An extensive survey failed to reveal a mid diakinesis stage and it was therefore not possible to demonstrate the presence of wheel-like structures in the chromatin bridges between the homologues. At late diakinesis, the confluent chromatin of the bivalents did not permit reconstructions of the individual bivalents of the complement, but in a number of cases, remnants of the dense core and the ring were found in what appeared to be chromatin bridges combining the homologues.

\subsubsection{Numbers of recombination nodules and chiasmata.}

The mean number of recombination nodules per nucleus at mid zygotene, late zygotene, early

Table VII

Mean number of recombination nodules $(R N)$ and chiasmata at early and mid diplotene in Coprinus meiocytes.

\begin{tabular}{lccccc}
\hline Stage & Cross & $\begin{array}{c}\text { Number of } \\
\text { nuclei }\end{array}$ & $\begin{array}{c}\text { Number of } \\
\text { RN } \pm \text { sd }\end{array}$ & $\begin{array}{c}\text { Number of } \\
\text { chiasmata } \pm \text { sd }\end{array}$ & $\begin{array}{c}\text { Total number of } \\
\text { RN and chiasmata } \pm \text { sd }\end{array}$ \\
\hline $\begin{array}{l}\text { Early } \\
\text { diplotene }\end{array}$ & JR $\times$ PR & 4 & $23 \pm 4$ & - & $23 \pm 4$ \\
\hline & $\mathrm{JR} \times \mathrm{E}$ & 4 & $24 \pm 2$ & - & $24 \pm 2$ \\
$\begin{array}{l}\text { Mid } \\
\text { diplotene }\end{array}$ & $\mathrm{JR} \times \mathrm{PR}$ & 2 & 17 & 11 & 28 \\
\hline
\end{tabular}


Table VIII

Mean number of recombination nodules (RN) and chiasmata during late meiotic prophase in Coprinus meiocytes.

\begin{tabular}{lcccc}
\hline Stage & $\begin{array}{c}\text { Number of } \\
\text { nuclei }\end{array}$ & $\begin{array}{c}\text { Number of } \\
\text { RN } \pm \text { sd }\end{array}$ & $\begin{array}{c}\text { Number of } \\
\text { chiasmata } \pm \text { sd }\end{array}$ & $\begin{array}{c}\text { Total number of } \\
\text { RN and chiasmata } \pm \text { sd }\end{array}$ \\
\hline Early diplotene & 8 & $23 \pm 3$ & - & $23 \pm 3$ \\
Mid diplotene & 6 & $13 \pm 9$ & $14 \pm 6$ & $27 \pm 3$ \\
Late diplotene & 9 & 0.4 & $23 \pm 4$ & $23 \pm 4$ \\
Early diakinesis & 1 & - & 24 & 24 \\
\hline
\end{tabular}

pachytene and mid-late pachytene is given in Tables I and II. As is the case for the mean complement length per nucleus, the mean number of nodules per nucleus is virtually identical for the three crosses. At early pachytene, the mean number of nodules amounts to 36,38 and 37 for the three crosses, the corresponding values for the two crosses examined at mid-late pachytene being 24 and 27 . As the differences between the three crosses were considered insignificant, the data were pooled in the following analysis of the nodule distribution. Although the scarcity of data for the zygotene stages does not allow a detailed comparison, there seems no a priori reason to expect differences at this stage considering the similarity of the pachytene data.

The pooled data (Table III) allow the following general conclusions: 1) The number of nodules expected upon completion of pairing and synaptonemal complex formation at late zygotene is identical to that observed at early pachytene, amounting to 37 recombination nodules per nucleus. 2) At mid-late pachytene, the mean number of nodules per nucleus is 26 , a $30 \%$ decrease of the number at late zygotene and early pachytene. 3) A similar reduction of the number of nodules per nucleus is observed between mid and late zygotene but since only three mid zygotene nuclei were reconstructed and due to the considerable internuclear variation in number of nodules per nucleus in zygotene as a whole, this reduction may reflect an insufficient number of observations.

Also at early and mid diplotene the number of nodules and chiasmata was very similar in the two crosses (Table VII), the sum of nodules and chiasmata being 23 in cross JR52 $\times$ PR2301 and 24 in cross JR52 $\times$ E991 at early diplotene and 28 and 27 at mid diplotene. Table VIII combines the pooled data for early and mid diplotene with the late diplotene and early diakinesis data from cross JR52 $\times$ PR2301. These results demonstrate that during diplotene all nodules present at mid-late pachytene are converted into chiasmata. The number of chiasmata then remains constant during late diplotene and at early diakinesis.

\subsection{Distribution of recombination nodules among bivalents}

The assessment of the distribution of recombination nodules among the bivalents of a nucleus at the different stages of the early meiotic prophase in Coprinus meiocytes was performed as previously described for human and Bombyx spermatocytes $(15,27)$. The frequency of bivalents with $0,1,2, \ldots, 7,>7$ nodules was determined for each stage and compared with the frequency one would expect if the nodules were distributed randomly among the bivalents. The translocation quadrivalents were not included in this analysis.

The expected random distribution was constructed by a computer simulation experiment. The absolute length and centromere index of the individual bivalents of each nucleus were fed into a Honeywell Bull H6000 computer together with the number of nodules of that particular nucleus, excluding the nodules of the quadrivalents. The computer was programmed to position the nodules, one at a time, at random among and along the bivalents as described previously (27). The program was run a total of 10,500 times for each stage, the number of runs for a given nucleus corresponding to 10,500 divided by the 
Table IX

Observed and expected random distributions of nodules among the bivalents in Coprinus meiocytes. $\mathrm{O}$, observed frequency; $T$, theoretical frequency.

\begin{tabular}{lcccccccccc}
\hline & \multicolumn{10}{c}{ Percent bivalents with n nodules } \\
\cline { 2 - 12 } Stage & $\mathrm{n}$ & 0 & 1 & 2 & 3 & 4 & 5 & 6 & 7 & $>7$ \\
\hline \multirow{2}{*}{ Late zygotene } & $\mathrm{O}$ & 8.1 & 33.7 & 20.9 & 12.8 & 12.8 & 3.5 & 4.7 & 2.3 & 1.2 \\
& $\mathrm{~T}$ & 13.7 & 22.8 & 22.5 & 17.1 & 11.2 & 6.4 & 3.5 & 1.6 & 1.1 \\
\hline \multirow{2}{*}{ Early pachytene } & $\mathrm{O}$ & 2.1 & 20.6 & 29.6 & 24.3 & 9.5 & 7.9 & 3.2 & 2.6 & 0 \\
& $\mathrm{~T}$ & 8.8 & 20.4 & 24.1 & 19.8 & 13.2 & 7.4 & 3.7 & 1.6 & 0.9 \\
\hline Mid-late & $\mathrm{O}$ & 1.7 & 32.5 & 41.6 & 18.2 & 3.5 & 1.7 & 0.5 & 0.5 & 0 \\
pachytene & $\mathrm{T}$ & 14.0 & 27.6 & 27.3 & 17.5 & 8.7 & 3.4 & 1.2 & 0.3 & 0.1 \\
\hline Early diplotenea & $\mathrm{O}$ & 0 & 50.0 & 29.5 & 18.2 & 2.2 & 0 & 0 & 0 & 0 \\
\hline
\end{tabular}

a) Data from four completely reconstructed early diplotene nuclei.

number of nuclei reconstructed for that stage. In this way, the expected frequencies of bivalents with $0,1,2, \ldots, 7,>7$ nodules were determined together with all neighbour nodule, nodule-centromere and nodule-telomere distances (see section 3.8). For the late zygotene nuclei, the unpaired stretches were excluded by joining the paired segments. Distances between neighbour nodules as well as nodule-telomere distances were not measured across the centromere. A comparison between the observed and expected frequency of bivalents with $0,1,2, \ldots, 7,>7$ nodules (Table IX) permits the following conclusions:

1) At late zygotene, the observed and expected frequencies are very similar, the only prominent exception being the frequencies of bivalents without nodules. Only $61 \%$ of the expected number of bivalents were devoid of nodules. This deficit is compensated for by a corresponding excess of bivalents with one nodule. It should be kept in mind, however, that the observed data only include bivalents from 8 nuclei.

2) At early pachytene, the observed and expected frequencies are likewise very similar, the major difference being in the frequency of bivalents without nodules, where $2.1 \%$ were observed but $8.8 \%$ expected. In contrast, biva- lents with 2 and 3 nodules were slightly more frequent than expected from a random distribution.

3) At mid-late pachytene, only $1.7 \%$ of the bivalents are devoid of nodules while $14 \%$ are expected. The reverse pattern is observed for bivalents with one and in particular two nodules.

Table IX also includes observed data from 4 early diplotene nuclei. At this stage, all bivalents possess one to four nodules, the group of bivalents with one nodule amounting to $50 \%$. When taken together, these data demonstrate that recombination nodules are distributed nearly at random during zygotene but that mechanisms exist which by altering the distribution tend to ensure a minimum of one nodule per bivalent.

\subsection{Distribution of recombination nodules along bivalents}

3.8.1. Distribution of nodules among the two arms of the bivalents

In order to investigate whether the placement of recombination nodules occurs independently in the two arms of the bivalents or whether the association of a nodule in one arm changes the probability for the attachment of a second nodule 
Table X

Observed and expected random distributions of recombination nodules among the arms of bivalents with 2,3 and 4 nodules. $O$, observed frequency; $T$, theoretical frequency.

\begin{tabular}{lcccccccc}
\hline & \multicolumn{7}{c}{ Percent nodules in arms of bivalents with n nodules ( $\mathrm{p}$ arm $+\mathrm{q}$ arm) } \\
\cline { 2 - 9 } & $\mathrm{n}=$ & $(0+2)$ & $(1+1)$ & $(0+3)$ & $(1+2)$ & $(0+4)$ & $(1+3)$ & $(2+2)$ \\
\hline Late & $\mathrm{O}$ & 68 & 32 & 33 & 66 & 30 & 50 & 20 \\
zygotene & $\mathrm{T}$ & 58 & 42 & 35 & 65 & 23 & 48 & 30 \\
\hline Early & $\mathrm{O}$ & 35 & 65 & 18 & 82 & 5 & 63 & 32 \\
pachytene & $\mathrm{T}$ & 57 & 43 & 35 & 65 & 23 & 48 & 30 \\
\hline Mid-late & $\mathrm{O}$ & 27 & 73 & 14 & 86 & 13 & 63 & 25 \\
pachytene & $\mathrm{T}$ & 55 & 45 & 33 & 67 & 21 & 48 & 31 \\
\hline
\end{tabular}

in the same arm, the number of bivalents with two three and four nodules was determined and compared with the numbers obtained by the computer simulation experiment. Bivalents with five or more nodules were not included in the analysis due to their low frequency. The results are presented in Table X. For bivalents with two nodules, the observed ratio of bivalents with both nodules in the same arm and bivalents with nodules in either arm are almost identical to the ratios based on the theoretical random distribution at late zygotene. (As described in section 3.7 , the computer simulation does take into account the fact that the individual bivalent arms are of different length.) Although based on a more limited number of observations, the distribution of nodules in the two arms of bivalents with three and four nodules is also very similar to that expected if nodules were positioned independently in the two arms of the bivalents.

The situation at early and mid-late pachytene is strikingly different (Table $\mathrm{X}$ ): among bivalents with two nodules, $35 \%$ and $27 \%$ had both nodules in the same arm at the two stages while the simulated random positioning resulted in $57 \%$ and $55 \%$ bivalents with both nodules in the same arm. The tendency of nodules to be more equally distributed among the two arms of a bivalent than can be accounted for alone by a random positioning, is also evident for bivalents with three and four nodules at early and mid-late pachytene.

These observations are in agreement with the observed decrease in bivalents without nodules after zygotene and furthermore show that placement of nodules on the bivalents is not a random process at pachytene but that arms without nodules more frequently receive a nodule than arms which already have one.

\subsubsection{Distribution of nodules on bivalent arms}

The normalization procedure performed on the arms of the chromosomes in the translocation quadrivalent, as outlined in section 3.2. was carried out in order to compensate for the internuclear variation in arm length before analyzing the distribution of recombination nodules along the individual bivalents. The number of nodules per bivalent segment for all nuclei at late zygotene, early pachytene and midlate pachytene is presented in histograms in Fig. ure 19, each segment corresponding to $0.4 \mu \mathrm{m}$ of synaptonemal complex after normalization. As outlined in section 3.4 , the identification of most of the bivalents beyond a division into four groups is ambiguous, exceptions being bivalents $I$ and 13. Hence, a detailed interpretation of the distribution of nodules along the bivalents is not warranted, and only the following general conclusions are considered relevant:

1) The relatively few late zygotene data 
LATE ZYGOTENE EARLY PACHYTENE MID-LATE PACHYT.
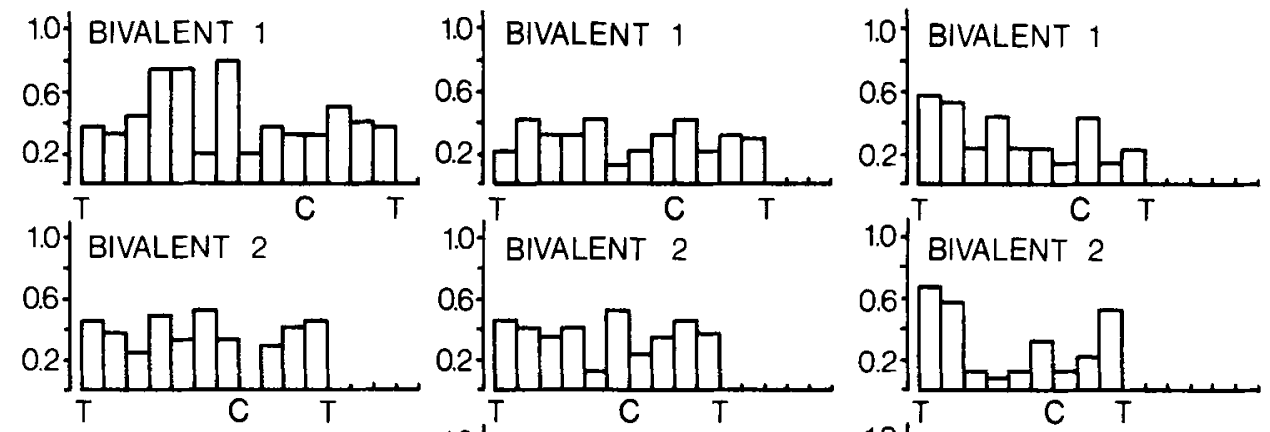

1.0. 'ं BIVALENT 2
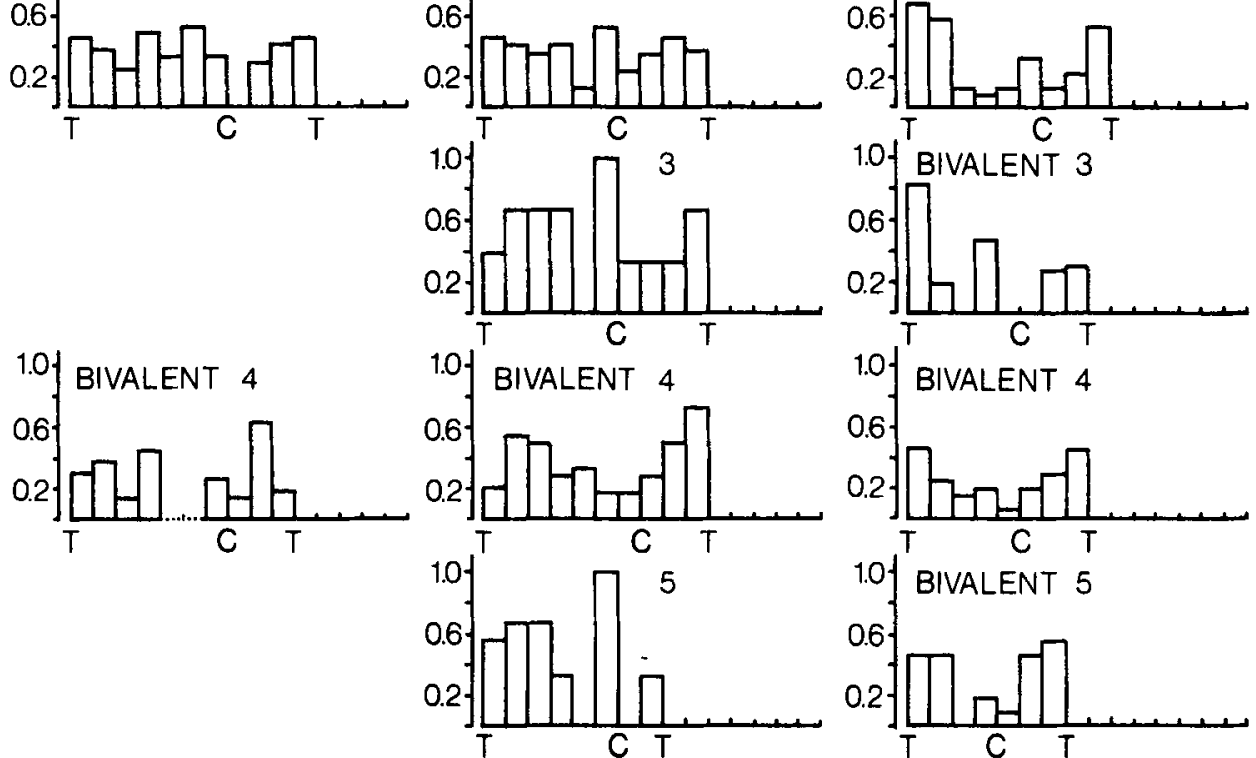

1.0. BIVALENT 4
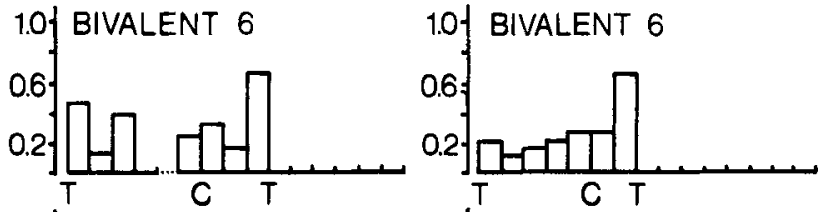

0.6 .
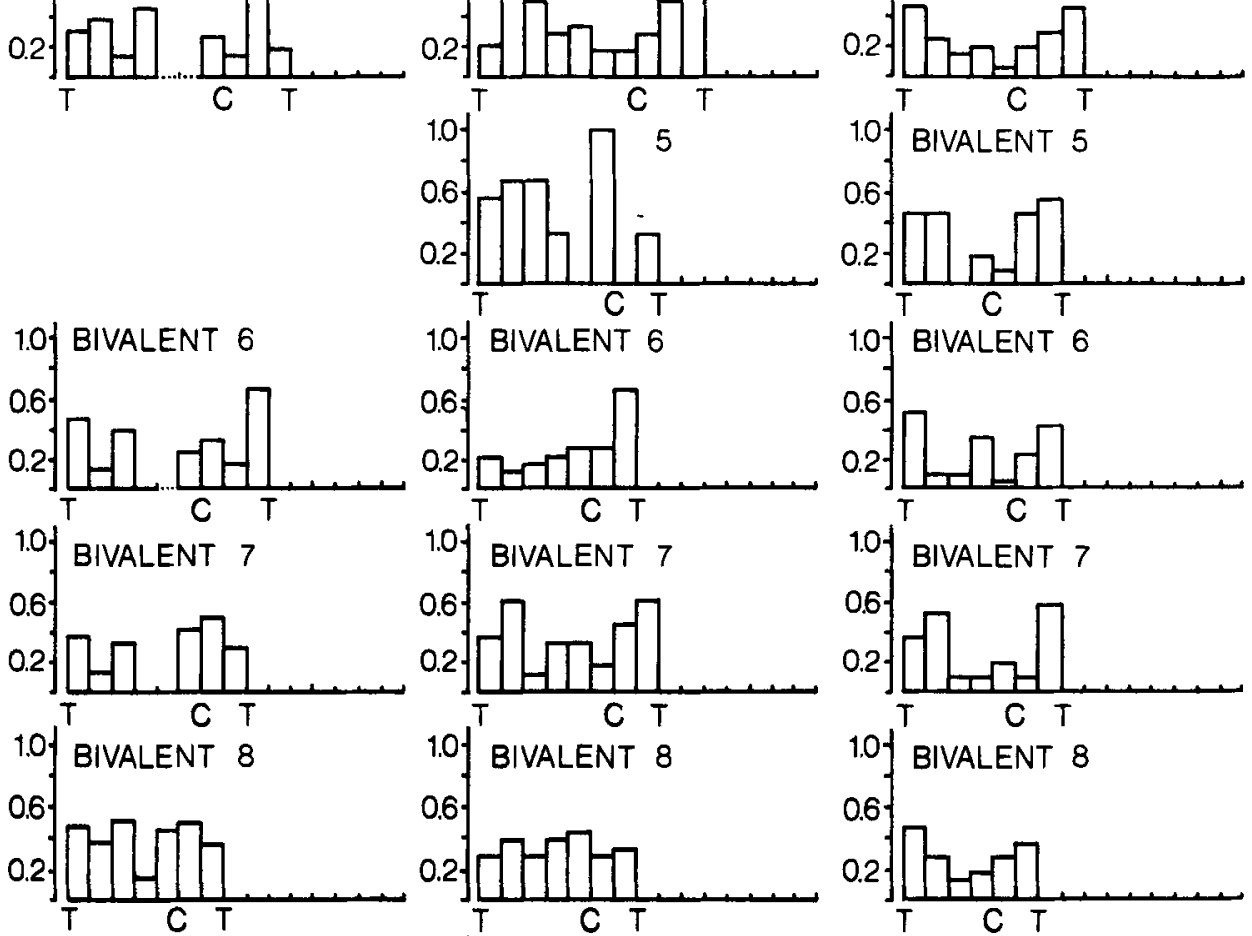

1.0 BIVALENT 6

0.6

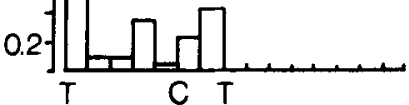

1.0 BIVALENT 7

0.6
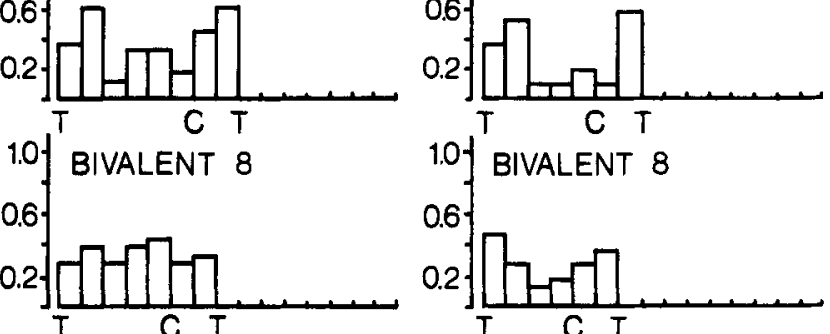

1.0 BIVALENT 8

0.6
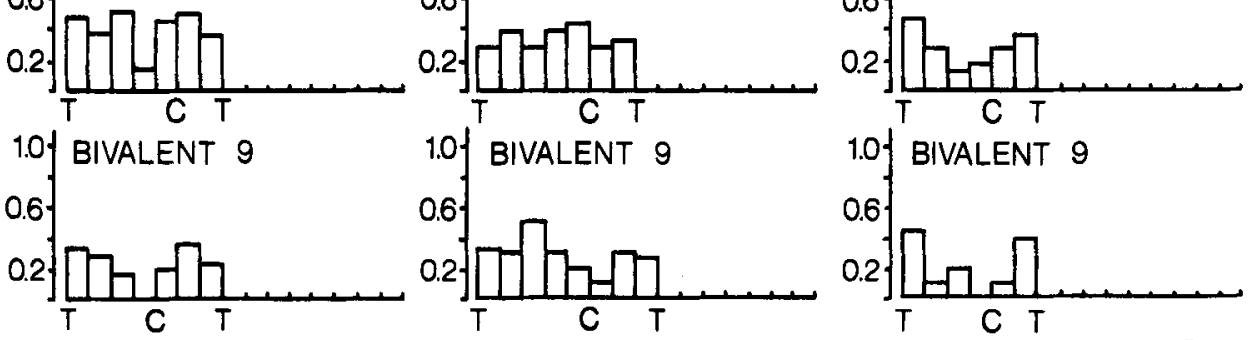

19 


\section{LATE ZYGOTENE EARLY PACHYTENE MID-LATE PACHYT.}
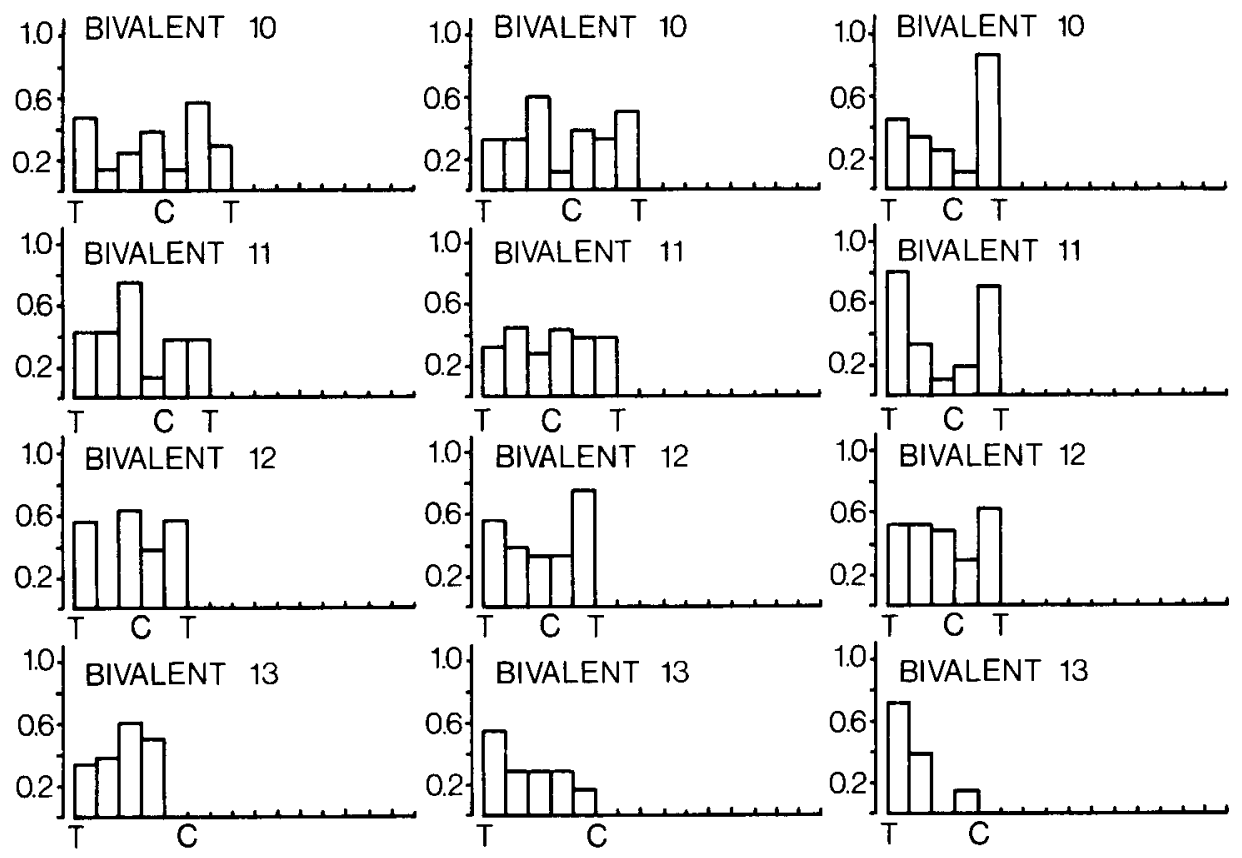

19

Figure 19. Histograms showing the distribution of recombination nodules on bivalents $1-13$ at late zygotene, early pachytene and mid-late pachytene.

The columns represent the frequencies of nodules per bivalent in $0.4 \mu \mathrm{m}$ segments of normalized bivalent arms. As all late zygotene nuclei contain translocation quadrivalent 1 consisting of chromosomes 3 and 5 , the nodule distribution in the arms of these bivalents is not included but shown in a separate diagram in Figure 21. The frequencies of nodules at early and mid-late pachytene along bivalents 3 and 5 are from cross 3 (with translocation 2) while the frequencies of nodules on bivalents 1 and 9 are from cross 2 (with translocation 1 ). For the remainder of the bivalents, data from all three crosses have been pooled.

suggest that nodules are distributed nearly at random along the bivalents. In some bivalents (e.g., bivalents 2, 4, 9, 10 and 11), a minor deficit of nodules around the centromere is observed, whereas an excess of nodules in the telomere regions is only seen in a few bivalents (e.g., bivalent 6).

2) The distribution of nodules at early pachytene is very similar to that at late zygotene, suggesting a basically random distribution of nodules along the bivalent.

3) At mid-late pachytene, the nodule distribution is drastically different. In nearly all bivalents, the frequency of nodules is low on either side of the centromere, while it is high in telomeric segments.
These observations clearly demonstrate that a change in nodule distribution along the bivalents occurs between early and mid-late pachytene. The nearly random distribution found at late zygotene and early pachytene changes at mid-late pachytene to a distribution where telomeric segments are favoured over the remainder of the bivalent while the centromere region is selectively depleted of nodules.

\subsubsection{Distribution of nodules relative to each other, to centromere and telomere regions}

In order to analyze the distribution of nodules along the bivalents in more detail, all neighbour nodule, nodule-centromere and nodule-telomere 


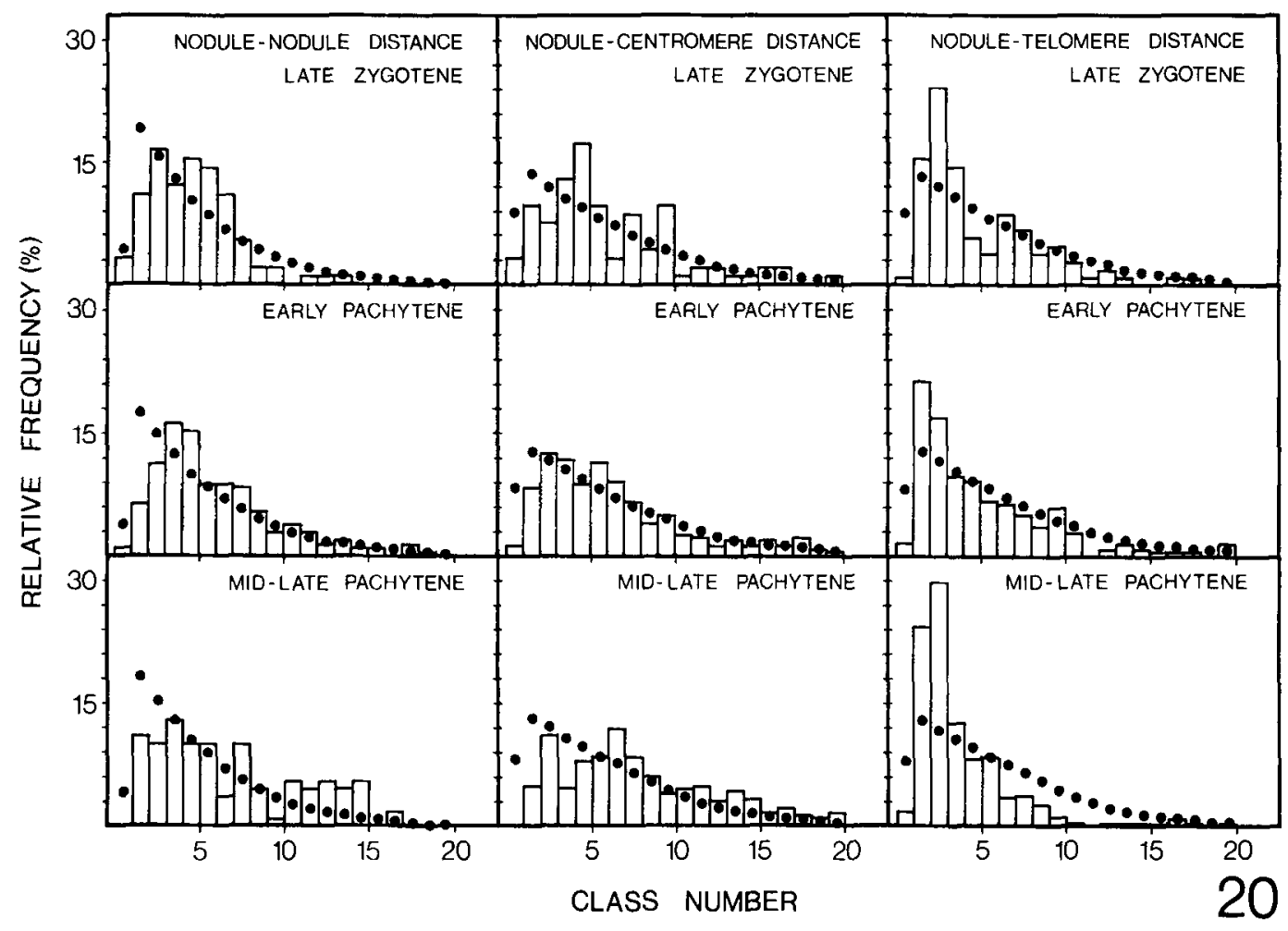

Figure 20. Histograms showing the distribution of nodule-nodule, nodule-centromere and nodule-telomere distances at late zygotene (LZ), early pachytene (EP) and mid-late pachytene (MLP).

The columns represent the relative frequencies of distances in $0.1 \mu \mathrm{m}$ classes from 0 to $2 \mu \mathrm{m}$. $(\mathrm{n}=90 \mathrm{LZ}$, $203 \mathrm{EP}, 110 \mathrm{MLP}$ for nodule-nodule distances; $\mathrm{n}=92 \mathrm{LZ}, 296 \mathrm{EP}, 343 \mathrm{MLP}$ for nodule-centromere distances; and $n=103 \mathrm{LZ}, 295 \mathrm{EP}, 339 \mathrm{MLP}$ for nodule-telomere distances.) The dotted lines are the expected random distributions of distances. $(\mathrm{n}=125,839 \mathrm{LZ}, 134,383 \mathrm{EP}, 86,694$ MLP for nodule-nodule distances; $\mathrm{n}=142$, $711 \mathrm{LZ}, 149,817 \mathrm{EP}$ 140, 306 MLP for nodule-centromere and nodule-telomere distances).

distances were measured for each stage and compared to the distances obtained by the computer simulation experiment. The following conclusions can be drawn from these compari. sons (Figure 20):

1) At all three stages, there is a deficit of the observed short neighbour nodule distances, a deficit which becomes more pronounced towards the end of pachytene. For late zygotene, early pachytene and mid-late pachytene $39 \%, 36 \%$ and $37 \%$ respectively of all distances are expected within the interval $0-0.3 \mu \mathrm{m}$ while the observed frequencies only amount to $31 \%, 19 \%$ and $21 \%$, In contrast, neighbour nodule distances ranging from 0.4 to $0.7 \mu \mathrm{m}$ at late zygotene, 0.3 to $0.5 \mu \mathrm{m}$ at early pachytene and 1.0 to 1.5 $\mu \mathrm{m}$ at mid-late pachytene were more frequent than expected from a random distribution.

2) At all three stages, nodules are less frequently observed than expected from 0 to $0.3 \mu \mathrm{m}$ from the centromere. For mid-late pachytene, this deficit is especially pronounced and includes also distances from 0.3 to $0.5 \mu \mathrm{m}$.

3) Nodules are positioned closer to the telomere than expected at all three stages, excluding the interval $0-0.1 \mu \mathrm{m}$. This pattern is especially pronounced at mid pachytene where $54 \%$ of the distances are within the interval from 0.2 to 0.4 $\mu \mathrm{m}$ against an expected frequency of $25 \%$.

It is thus apparent that nodules, in particular at early and mid-late pachytene, are farther apart than expected. Furthermore nodules are closer to 

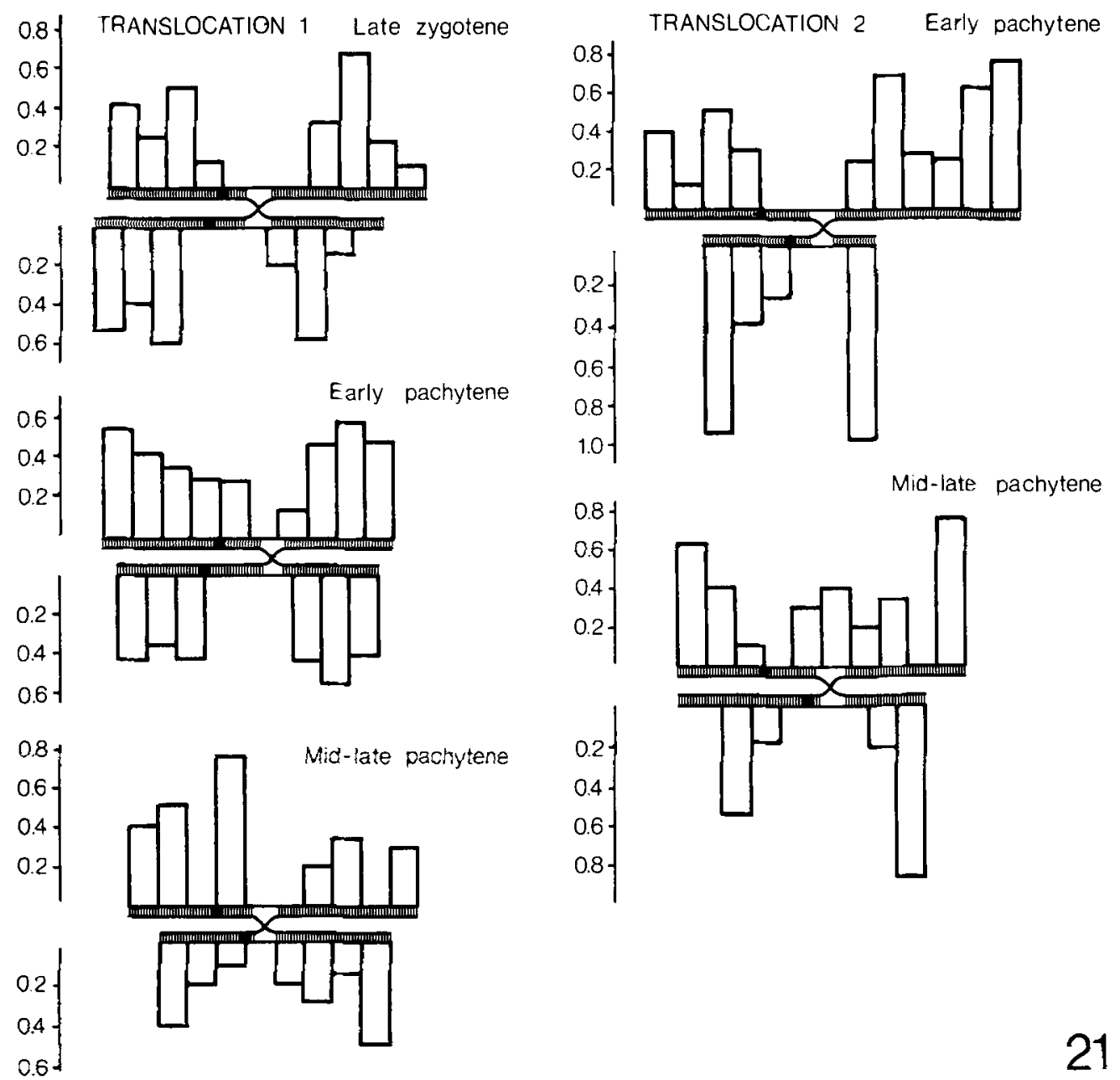

Figure 21. Diagrams showing the position of pairing partner exchange and the frequency of recombination nodules in $0.4 \mu \mathrm{m}$ intervals along the four arms of translocation quadrivalents 1 and 2

The filled circles denote the position of the centromere regions.

the telomere and farther away from the centromere than expected. This is especially pronounced at mid-late pachytene, and is in agreement with the conclusion inferred from the analysis of the distribution of nodules along the bivalents (see section 3.8.2).

\subsection{Distribution of recombination nodules within the translocation quadrivalent}

The distribution of recombination nodules within the translocation quadrivalents does not appear to deviate from that observed in the remainder of the complement. As seen in Figure 21, nodules are in excess at the telomere regions at early and mid-late pachytene in both translocation quadrivalents whereas centromere regions are relatively depleted of nodules. The distribution of nodules in chromosome regions flanking the site of pairing partner exchange was not analyzed as these regions were too often unpaired to provide sufficient observations. The normalized arms of the translocation quadrivalents did not, however, reveal any major accumulation or depletion of nodules in the synaptonemal complex segments fanking the unpaired regions, when compared to neighbouring segments.

In addition, the distribution of recombination 


\begin{tabular}{|c|c|c|}
\hline STAGE & \begin{tabular}{|c|} 
EARLY \\
PACHYTENE
\end{tabular} & $\begin{array}{l}\text { MID - LATE } \\
\text { PACHYTENE }\end{array}$ \\
\hline 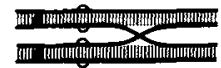 & 1 & 0 \\
\hline 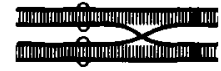 & 1 & 0 \\
\hline 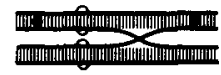 & 1 & 1 \\
\hline 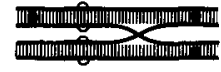 & 6 & 5 \\
\hline 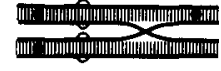 & 0 & 1 \\
\hline 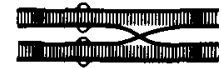 & 10 & 6 \\
\hline
\end{tabular}

22

Figure 22. Diagrams and table showing the position of recombination nodules relative to the site of pairing partner exchange in the translocation quadrivalents

The total number of quadrivalents is given for each category at early and mid-late pachytene.

nodules among the four arms of the translocation quadrivalents was analyzed. At mid-late pachytene, all except one quadrivalent possessed nodules in three or all four arms. In the arms of the translocations, nodules were present distal to the site of pairing partner exchange (Figure 22). The remaining quadrivalent contained only two nodules, one distal to the exchange site and one in the opposite arm. At early pachytene, nodules were located proximal to the site of pairing partner exchange in two translocation quadrivalents, while the remaining quadrivalents all had nodules in three or all four regions flanking the exchange site. The implications of these distributions are discussed in section 4.1.

\section{DISCUSSION}

\subsection{Chromosome pairing}

\subsubsection{Translocation chromosomes and their ho- mologues}

The present study has unequivocally demonstrated the presence of one translocation in each of the strains PR2301 and E991. In the former strain, the translocation involves chromosomes 3 and 5 , and in the latter chromosomes 1 and 9.

The specific pairing and synaptonemal complex formation in the translocated chromosomes and their homologues during zygotene have resulted in the formation of translocation quadrivalents in most nuclei. Despite substantial internuclear differences in the position of the site of pairing partner exchange, the average position of the exchange site is about the same at late zygotene, early and mid-late pachytene. In addition to these regularly paired translocation quadrivalents, a number of apparent quadrivalents, but with discontinuous lateral components in the exchange region have been observed. Finally, the translocation quadrivalent was replaced in some nuclei by two bivalents.

These observations are consistent with the mechanism of chromosome pairing found in tetraploid Bombyx oocytes: quadrivalents regularly form during the specific pairing phase in zygotene but are in the recombination deficient female subsequently transformed into bivalents by dissolution and reassembly of the central component of the synaptonemal complex. In contrast, crossing over and chiasma formation in tetraploid Bombyx males prevent the transformation of quadrivalents into bivalents (28). The existence of this correction system for chromosome pairing which has not resulted in bivalent formation and the effect of crossing over on the correction process has furthermore been inferred from observations in triploid Bombyx (26), in human spermatocytes heterozygous for a reciprocal translocation (13) and recently in hexaploid wheat $(10)$.

The presence of two heteromorphic bivalents (of which one was broken, Figure 16c) in a single mid-late pachytene nucleus unambiguously demonstrates that transformation of multivalents into bivalents occurs also in Coprinus. The very low number of nuclei in which the translocation quadrivalent is replaced by two bivalents is in agreement with the observed distribution of recombination nodules in the four arms of the translocation quadrivalents (section 3.9 and Figure 22) and confirms the inhibitory effect of crossing over on correction of chromosome pairing. Transformation of the quadrivalent into two heteromorphic bivalents by partial dissolu- 
tion and reassembly of the central region of the complex is theoretically possible in only two of the early pachytene nuclei and in none of the mid-late pachytene nuclei if regions containing recombination nodules act as barriers to the dissolution. As discussed in section 4.5, centromere regions may assert a similar effect on the stability of chromosome associations and may in combination with the observed nodule distribution account for the preservation of the quadrivalent configuration in the vast majority of nuclei analyzed.

The frequency of lateral component discontinuity is remarkably high in the translocation chromosomes and their homologues. In all analyzed mid zygotene and in $30 \%$ of the late zygotene nuclei, one or more of the lateral components are discontinuous. Most likely, these discontinuities reflect chromosome breaks associated with resolution of previously interlocked chromosome regions. As such breaks have not been observed in the 20 early pachytene nuclei analyzed, the continuity of the four chromosomes of the translocation quadrivalent is reestablished by reunion of the broken chromosome ends. One important exception to this general pattern is revealed, however, in a single nucleus where a translocation quadrivalent could not be identified. Instead, two apparently normal bivalents, i.e., with continuous lateral components of exactly the same length, were formed.

Unless extensive "synaptic adjustment « (22, 23) has occured in originally heteromorphic bivalents, originating from correction of a translocation quadrivalent by dissolution and reassembly of the central region of the synaptonemal complex, the presence of two apparently homomorphic bivalents signifies that the breakreunion mechanism, responsible for correction of interlockings, in rare cases leads to »retranslocation «. As shown in Figure 23, breakage in either of the two translocation chromosomes at or near the original break points, or in the two normal chromosomes at the corresponding sites, can lead to homozygosity for either configuration in cases where the second break coincides with the primary. In cases of a slightly different location of the second break, heterozygosity for minor deficiencies and duplications would result.

In one early and six mid-late pachytene nuclei,

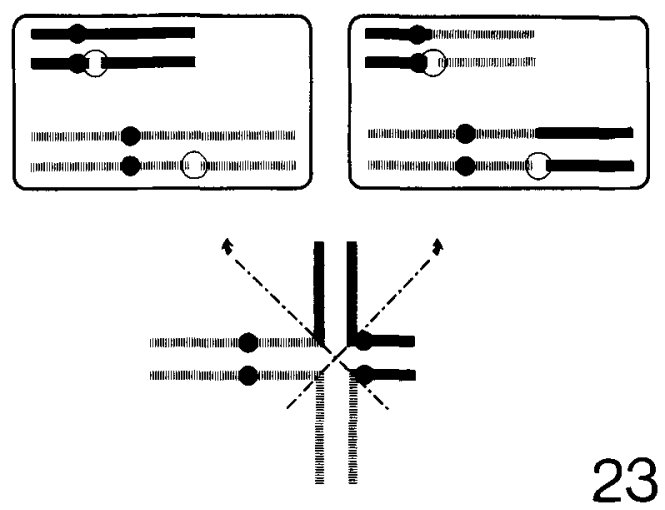

Figure 23. Diagram illustrating the proposed transformation of a translocation quadrivalent into two bivalents by a breakage-reunion mechanism.

quadrivalents could not be unequivocally identified, the translocation and normal chromosomes being recognizable only by a $100-200 \mathrm{~nm}$ disruption of one lateral component of otherwise normally paired bivalents. Although chromatin continuity between the two "bivalents « cannot be excluded, it is conceivable that these configurations represent intermediates in the $\gg$ correction « of the translocation quadrivalents by a breakage-reunion process as described above.

\subsubsection{Chromosome pairing in nontranslocation chromosomes}

The present results on chromosome pairing in Coprinus meiocytes conform with the general scheme for chromosome pairing established through three dimensional reconstructions of whole nuclei in a variety of organisms (29). Pairing and synaptonemal complex formation are generally initiated from the telomeres but may also be initiated interstitially. Interlocking of chromosomes and bivalents occurs during pairing and synaptonemal complex formation at zygotene and is resolved at zygotene-pachytene by breakage-reunion of the interlocked chromosomes and bivalents as is the case in Homo (27), Bombyx (15) and Triticum (10) and probably also Sordaria (32). In two of the four cases of interlocking at zygotene, the translocation quadrivalent has interlocked other chromosomes and bivalents, whereas the remaining two cases are interlockings of two bivalents or a bivalent and one of the quadrivalent arms. Hence, 
interlockings frequently involve the translocation chromosomes but occur also at a lower frequency with normal bivalents. Apparently, the shorter chromosomes of Coprinus interlock less frequently than the two- to threefold longer chromosomes of Homo and Bombyx, where $60 \%$ and $100 \%$ of the late zygotene nuclei respectively contain one or more interlockings. A total of $44 \%$ of the zygotene nuclei contain chromosome aberrations in the form of breaks and interlockings, i. e., considerably fewer than the $80 \%$ and $100 \%$ of nuclei with aberrations seen in Homo and Bombyx. An even lower frequency of aberrations would be expected in Coprinus strains without translocations.

These results from Coprinus are in accordance with observations in Sordaria humana $(n=7$, $\mathrm{SC}$ length $=40.2 \mu \mathrm{m}$ ) and Sordaria macrospora $(\mathrm{n}=7, \mathrm{SC}$ length $=58.0 \mu \mathrm{m})$. In the first species only one chromosome interlocking and one bivalent break were found in two zygotene and ten pachytene nuclei (31) while the reconstruction of six zygotene and three pachytene nuclei from the second species did not reveal any aberrations (32).

It thus appears that the frequency of interlocking and chromosome breakage are related to the chromosome number as well as complement length, few and short chromosomes decreasing the risk of interlocking during chromosome pairing at zygotene.

As is the case in all other organisms investigated so far (29), pairing and synaptonemal complex formation are highly specific during zygotene in Coprinus meiocytes. Only one case of nonhomologous pairing and synaptonemal complex formation has been observed, the free ends of a broken lateral component being paired with a short stretch of central region. Hence, the present analysis constitutes another example in support of the contention that nonhomologous pairing and synaptonemal complex formation during zygotene occur only in rare cases and is nearly always confined to interlocked or broken chromosome regions (15).

At early pachytene, all interlockings except one and all chromosome breaks have disappeared as is also the case in Homo, Bombyx and Triticum where only bivalent breaks are sometimes left unrepaired. At mid-late pachytene neither interlockings nor breaks, other than the possible breaks in the translocation quadrivalents, were found.

Two cases of nonhomologous pairing were found at mid-late pachytene (section 3.5). In addition, one of these configurations had an apparently normal synaptonemal complex in an unpaired chromosome region. The analysis of chromosome pairing in triploid Coprinus (30) revealed in a number of cases the same phenomenon which unambiguously demonstrates that normal appearing synaptonemal complexes can assemble also in cases where only a segment of one lateral component is available.

\subsection{Recombination nodules and chiasmata}

The first appearance of recombination nodules concomitant with the initiation of pairing and synaptonemal complex formation, the subsequent morphological evolution of nodules, as well as the fluctuations in the number of nodules during the meiotic prophase, is consistent with the pattern observed in Homo (27) and Bombyx (15). Also, the presence of nodules associated with precursor material for the central region at the pairing fork further reinforces the contention that nodules are prerequisites for rather than the result of a recombination event. Conclusive evidence for the latter point is presented in the report on meiosis in triploid Coprinus meiocytes (30) in which it is shown that nodules in several cases are present in central region material associated with unpaired lateral components.

The gradual reduction in the number of nodules per nucleus which takes place during zygotene and pachytene in Coprinus meiocytes, confirms that this phenomenon is a general feature of meiosis: the reduction amounts to $30 \%$ in Coprinus and is thus of the same order of magnitude as the reduction observed in Schizophyllum - $41 \%$, human spermatocytes $48 \%$ and Bombyx spermatocytes - $41 \%$, although the decrease occurs at slightly different periods relative to the completion of synaptonemal complex formation. This reduction has been suggested to relate to the occurrence of nonreciprocal recombination $(15,27)$, mainly based on the assumption that reciprocal and nonreciprocal recombination are mediated by similar or identical enzyme systems, the outcome being alone dependent on how the proposed intermedi- 
ate - the half chiasma- is resolved $(6,11)$. Hence, elimination of a given nodule could signify the termination of a nonreciprocal event, whereas reciprocal recombination and the subsequent formation of a physically stable chiasma require that the nodule remain at the site of the crossover. This proposal implies that the processes resulting in crossing over and gene conversion are initiated during zygotene and that most of the gene conversion events are completed during early pachytene.

Recombination nodules increase in size and density during pachytene in Coprinus, Homo and Bombyx. The subsequent transformation of recombination nodules into more loose chromatin-like structures is similar to that observed in Bombyx spermatocytes. In both organisms, these small chromatin condensations - chromatin nodules - remain associated with remnants of the synaptonemal complex during early and mid diplotene, and by late diplotene, when synaptonemal complex elimination is nearly completed, the chromatin nodules have developed into more elaborate chiasma structures. As in Bombyx, the number of recombination nodules at late pachytene is almost identical to the sum of nodules and chiasmata through diplotene up to early diakinesis. In both organisms, the mid diplotene - early diakinesis chiasma consists of a mass of differentially condensed chromatin forming a ringlike structure. In Bombyx, this structure is flanked by two regions of compacted chromatin, while this is not the case in Coprinus. As nuclei in mid diakinesis were not found in the present study it was not possible to show directly whether the chromatin bridges between the homologous chromosomes at mid diakinesis contained morphologically identifiable structures similar to those characteristic of late diplotene early diakinesis nuclei.

These observations provide additional evidence for the proposal (15) that chiasmata originate from bivalent regions which at late pachytene contain recombination nodules and that the nodule itself and probably also constituents of the synaptonemal complex participate in the organization of the elaborate chiasma.

\subsection{Distribution of recombination nodules among bivalents}

The change from late zygotene to mid-late pachytene in the number of bivalents with $0,1,2$. ... nodules relative to the numbers expected if nodules were distributed randomly among the bivalents give the following pattern: The initial random distribution gradually changes after zygotene resulting at mid-late pachytene in a drastic decrease from $8.1 \%$ to $1.7 \%$ in the frequency of bivalents without nodules. This is in agreement with observations in Bombyx and man and reinforces the contention that a modification of the nodule distribution, which tends to decrease the number of bivalents without nodules is a general feature of meiosis.

Despite the reduction in the number of bivalents without nodules, $1.7 \%$ of the bivalents in Coprinus are still devoid of nodules at midlate pachytene. If this implies that crossing over and chiasma formation will not occur in these bivalents, $0.9 \%$ nondisjunction is expected, resulting in a maximum of $10 \%$ unbalanced gametes. This calculation is based only on the 11 bivalents in the nucleus and does not include the translocation quadrivalent. In Bombyx spermatocytes the corresponding frequency amounted to $28 \%$ unbalanced gametes, when based on mid and late pachytene frequencies of bivalents devoid of nodules. It is not known whether these numbers are realistic, since the frequencies of primary nondisjunction are not known in these organisms. Two observations indicate that in Coprinus this estimate is too high: 1) The presence of at least one nodule on all bivalents in the four analyzed early diplotene nuclei indicates that the redistribution of nodules is not yet com-. pleted by mid-late pachytene. 2) Homologous centromere regions remain associated after the elimination of the synaptonemal complex at least to early diakinesis in Coprinus. The latter observation suggests that the physical contact of homologous centromere regions could increase the probability of a regular disjunction in bivalents without nodules/chiasmata by substituting for the action of chiasmata in preserving the association of the homologues up to metaphase I in such bivalents. 


\subsection{Distributions of recombination nodules along bivalents}

The distribution of nodules along bivalents was analyzed from three different angles in an attempt to reveal whether: 1) the two arms of a bivalent act as independent units with respect to their aquisition of nodules or if there is an interaction between the two bivalent arms, 2) different regions of the bivalents have equal affinity for nodules and 3) the attachment of one nodule to a bivalent influences the probability for the aquisition of a second nodule in the immediate vicinity of the first.

The analysis in Coprinus of the nodule distribution among the arms of a bivalent, the number of nodules in segments along bivalent arms as well as the distribution of neighbour nodule distances reveals at late zygotene a close resemblance between the observed distribution and that expected if nodules are positioned at random.

The same analyses, when performed on nuclei at mid-late pachytene, reveal fundamental differences between the observed and the constructed random distributions: among the bivalents with two or more nodules, there is an excess of bivalents with nodules in both arms. Within bivalent arms, nodules are more often located in segments near the telomeres than expected while they are relatively scarce in regions near the centromere. Finally, neighbour nodules are farther apart than expected.

These results are very similar to those obtained with the same procedures in Schizophyllum, Homo and Bombyx meiocytes. An excess of nodules close to the telomeres has been reported in the ascomycetes Sordaria macrospora (31) and Sordaria humana (32) while nodules are less prevalent in telomere regions in Neurospora (9). Recombination nodules are also distributed at random among and along the different bivalents at early pachytene in Drosophila while at mid-late pachytene a shift to a nonrandom distribution is accompanied by the appearence of a morphologically different type of nodule $(4,5)$.

On the basis of observations in Drosophila, Carpenter (5) proposed that the information for the placement of a nodule is embodied in the nodule itself. In view of the dynamic nature of the process of nodule placement, now known in
Homo, Bombyx and Coprinus, this proposal appears less attractive. A different explanation, is that the attachment of a nodule to a bivalent arm somehow decreases the arms affinity for additional nodules. This decrease could arise in two fundamentally different ways: either the nodule, after having associated with the central region of the complex, establishes a domain within which additional nodules cannot attach (or are selectively removed) or the central region of an entire chromosome arm becomes modified in a way as to reduce the affinity for additional nodules.

In an attempt to discriminate between these two possibilities, the following computer simulation experiment was performed on the mid-late pachytene data: as in the previous simulation experiments, the absolute bivalent lengths, centromere indices and total number of nodules for each nucleus were fed into the computer. The diameter of the nodules was, as before, $0.08 \mu \mathrm{m}$ at the time when a nodule was placed on a bivalent arm. The computer was then programmed to increase the diameter of the attached nodule before placing the next nodule, i. e., to establish a domain within which new nodules could not attach. In cases where a new nodule was to be placed within the domain of a previously positioned one it was rejected and a new position was generated. With respect to telomeres and centromeres, the minimum distance from the center of the nodule remained $0.4 \mu \mathrm{m}$ as in previous experiments. By stepwise increasing the size of the domain of attached nodules it was possible to determine a domain size which resulted in distributions of nodules among bivalents as well as between the two arms of the same bivalent which were fairly similar to those observed at mid-late pachytene (Table XI). This occurred at a diameter of the domain of attached nodules between 1.2 and $2.5 \mu \mathrm{m}$. Domains within this range drastically change the distribution of neighbour nodule distances and are obviously incompatible with the observed distribution of neighbour nodule distances at mid-late pachytene (Figure 20). Fifty-two and $80 \%$ of all neighbour nodule distances were observed within the intervals $0-0.6 \mu \mathrm{m}$ and $0-$ $1.2 \mu \mathrm{m}$ respectively while the computer simulation for nodule diameters of 1.2 and $2.5 \mu \mathrm{m}$ yielded only neighbour nodule distances larger 


\section{Table XI A}

The effect of creating stepwise increasing domains around a nodule of $0.08 \mu \mathrm{m}$ within which additional nodules cannot be placed. The distribution of nodules among bivalents at mid-late pachytene is shown. In all simulation experiments the domain is created as soon as a nodule has been positiontd.

\begin{tabular}{llrrrrrr}
\hline & \multicolumn{5}{c}{ Percent bivalents with n nodules } \\
\cline { 3 - 7 } & & $\mathrm{n}=$ & 0 & 1 & 2 & 3 & $>3$ \\
\hline Observed frequency & & 2 & 32 & 42 & 18 & 6 \\
\hline & & 14 & 28 & 27 & 17 & 14 \\
Theoretical frequen- & $0.08 \mu \mathrm{m}$ & 13 & 28 & 29 & 18 & 12 \\
cies for nodule domain & $0.24 \mu \mathrm{m}$ & 10 & 28 & 32 & 20 & 10 \\
diameters of: & $1.20 \mu \mathrm{m}$ & 5 & 27 & 40 & 22 & 6 \\
& $2.48 \mu \mathrm{m}^{\mathrm{a}}$ & 2 & 24 & 61 & 13 & 0 \\
\hline
\end{tabular}

a) The values are based on only 17 of the 21 mid-late pachytene nuclei as the large numbers of nodules in four nuclei $(24,24,28,32)$ could not be fitted in the synaptonemal complex complement.

\section{Table XI B}

The effect of creating stepwise increased exclusion domains around newly attached nodules on the distribution of nodules among bivalent arms at mid-late pachytene.

\begin{tabular}{|c|c|c|c|c|c|c|}
\hline & & \multicolumn{5}{|c|}{$\begin{array}{l}\text { Percent nodules in arms of bivalents } \\
\text { with n nodules ( } \mathrm{arm}+\mathrm{q} \text { arm) }\end{array}$} \\
\hline & & $\mathrm{n}=$ & $(0+2)$ & $(1+1)$ & $(0+3)$ & $(1+2)$ \\
\hline Observed frequencies & & & 27 & 73 & 14 & 86 \\
\hline $\begin{array}{l}\text { Theoretical frequen- } \\
\text { cies for nodule domain } \\
\text { diameters of: }\end{array}$ & $\begin{array}{l}0.08 \mu \mathrm{m} \\
0.24 \mu \mathrm{m} \\
0.56 \mu \mathrm{m} \\
1.20 \mu \mathrm{m} \\
2.48 \mu \mathrm{m}^{\mathrm{a}}\end{array}$ & & $\begin{array}{r}55 \\
52 \\
44 \\
27 \\
6\end{array}$ & $\begin{array}{l}45 \\
48 \\
56 \\
73 \\
94\end{array}$ & $\begin{array}{r}33 \\
29 \\
20 \\
7 \\
1\end{array}$ & $\begin{array}{l}67 \\
71 \\
80 \\
93 \\
99\end{array}$ \\
\hline
\end{tabular}

a) See Table XI A.

than 0.6 and $1.2 \mu \mathrm{m}$ respectively (Table XIII). This excludes the possibility that the nodule distribution at mid-late pachytene to any major extent is due to nodule-nodule interactions. More likely then, the observed distribution is the result of an interaction between the first nodule and the synaptonemal complex resulting in a decreased affinity of the bivalent arm for an additional placement of nodules while the position at which a second nodule attaches to a bivalent arm is only to a minor extent affected by the position of the first nodule. As illustrated in Table XII, the simulation of the action of such a mechanism, assuming a probability of 0.2 for placement of an additional nodule on an arm already carrying one, produces a distribution of bivalents with 0 , $1,2,3$ and $>3$ nodules very similar to that observed. Also the distribution of nodules among the arms of bivalents with 2 and 3 nodules becomes fairly similar to the observed one. Unlike the domain simulation (Table XII), this is achieved without significantly changing the distribution of neighbour nodule distances (Table XIII). As the distribution of recombination nodules at late zygotene and early pachytene is nearly random, this decreased affinity must 
Table XII A

The effect of a decreased affinitya of bivalent arms for the placements of a second and additional nodules on the distribution of nodules among bivalents at mid-late pachytene.

\begin{tabular}{lccccccc}
\hline & \multicolumn{6}{c}{ Percent bivalents with n nodules } \\
\cline { 2 - 7 } & $\mathrm{n}=$ & 0 & 1 & 2 & 3 & $>3$ \\
\hline Observed frequency & & 2 & 32 & 42 & 18 & 6 \\
\hline $\begin{array}{l}\text { Probability for attachment of } \\
\text { additional nodules }=0.2\end{array}$ & 4 & 27 & 44 & 19 & 6 \\
\hline
\end{tabular}

a) Nodules are initially positioned at random. In cases where a nodule is to be positioned on a bivalent arm which has already got one, a random number generator ensures that the second nodule is only positioned in one out of five cases. If the nodule is rejected, a new position is generated by the computer.

\section{Table XII B}

The effect of a decreased affinitya of bivalent arms for the placement of a second and additional nodules on the distribution of nodules among bivalent arms at mid-late pachytene.

\begin{tabular}{lccccc}
\hline & \multicolumn{5}{c}{$\begin{array}{c}\text { Percent nodules in arms of bivalents } \\
\text { with } \mathrm{n} \text { nodules }(\mathrm{p}+\mathrm{q} \text { arm })\end{array}$} \\
\cline { 2 - 7 } & $\mathrm{n}=$ & $(0+2)$ & $(1+1)$ & $(0+3)$ & $(1+2)$ \\
\hline Observed frequencies & & 27 & 73 & 14 & 86 \\
\hline $\begin{array}{l}\text { Probability for attachment of } \\
\text { additional nodules }=0.2\end{array}$ & 16 & 84 & 7 & 93 \\
\hline
\end{tabular}

a) See Table XII A.

\section{Table XIII}

Observed and computer simulated distributions of neighbour nodule distances: Percent distances in $0.1 \mu \mathrm{m}$ classes from 0 to $1.5 \mu \mathrm{m}$.

\begin{tabular}{|c|c|c|c|c|c|c|c|c|c|c|c|c|c|c|c|c|}
\hline \multirow{2}{*}{ Type of dataa } & \multicolumn{16}{|c|}{ Class number } \\
\hline & 1 & 2 & 3 & 4 & 5 & 6 & 7 & 8 & 9 & 10 & 11 & 12 & 13 & 14 & & $>15$ \\
\hline Observedb & 0 & 11 & 10 & 13 & 10 & 10 & 4 & 10 & 5 & 1 & 5 & 5 & 5 & 5 & 5 & 7 \\
\hline Randomc & 4 & 18 & 15 & 13 & 10 & 9 & 7 & 5 & 4 & 3 & 3 & 2 & 2 & 1 & 1 & 3 \\
\hline Domain $1.2 \mu \mathrm{m}^{\mathrm{d}}$ & 0 & 0 & 0 & 0 & 0 & 0 & 14 & 20 & 16 & 12 & 9 & 7 & 6 & 4 & 3 & 9 \\
\hline Domain $2.5 \mu \mathrm{me}$ & 0 & 0 & 0 & 0 & 0 & 0 & 0 & 0 & 0 & 0 & 0 & 0 & 6 & 24 & 18 & 52 \\
\hline Probability $0.2^{\mathrm{f}}$ & 3 & 16 & 14 & 12 & 10 & 9 & 7 & 6 & 5 & 4 & 3 & 3 & 2 & 2 & 1 & 3 \\
\hline
\end{tabular}

a) The experimental details are given in the text.

b) $n=110 ;$ c) $n=86,694$; d) $n=53,738 ;$ e) $n=16,221$; $n=47,327$. 
evolve after the initial positioning of nodules and affect only the secondary modification of the nodule distribution occurring after early pachytene.

Chiasma interference (position interference) may thus be the result of 1) a limited number of nodules available and 2) mechanisms which ensure at least one crossover/chiasma per bivalent as is required for its regular disjunction. As suggested above by the simulation experiment a main factor may be a reduced affinity for nodule placement once a bivalent (or bivalent arm) has got one nodule. Hence, only very few nodules are available for double crossovers within the same bivalent arm. If the majority of the nonreciprocal exchanges occurs during zygotene and early pachytene, where the distribution of nodules among and along the bivalents is nearly random, interference is not expected for nonreciprocal exchanges in accordance with the observed absence of such interference in numerous genetic analyses.

\section{ACKNOWLEDGEMENTS}

We would like to thank prof. D. vON WETTSTEIN and dr. J. G. BERTHELSEN for their critical review of the manuscript. The work was financially supported by grants 202-76-1 BIO DK and BIO-E-417 DK(G) from the Commission of the European Communities to professor D. VON WetTSTEIN, by grant A4693 from the Natural Science and Engineering Research Council of Canada to B. C. Lu and by grant LA 86 CNRS to D. ZiCKLeR.

\section{REFERENCES}

1. Byers, B. \& L. Goetsch: Electron microscopic observations on the meiotic kayrotype of diploid and tetraploid Saccharomyces cerevisiae. Proc. Nat. Acad. Sci. USA 72, 5056-5060 (1975)

2. Carmi, P., P. B. Holm, S. W. Rasmussen, J. SaGE \& D. ZICKLER: The pachytene karyotype of Schizophyllum commune analyzed by three dimensional reconstructions of synaptonemal complexes. Carlsberg Res. Commun. 43, $117-$ 132 (1978)

3. Carpenter, A. T. C.: Electron microscopy of meiosis in Drosophila melanogaster II. The recombination nodule - a recombination asso- ciated structure at pachytene? Proc. Nat. Acad. Sci. USA 72, 3186-3189 (1975)

4. Carpenter, A. T. C.: Synaptonemal complex and recombination nodules in wild type Drosophila melanogaster females. Genetics 92, 511541 (1979)

5. Carpenter, A. T. C.: Synaptonemal complex and recombination nodules in recombination deficient mutants of Drosophila melanogaster. Chromosoma (Berl.) 75, 259-292 (1979)

6. Catcheside D. G.: The genetics of recombination. In: Genetics Principles and Perspectives. K. R. Lewis \& B. John eds. Edward Arnold. London Vol. 2 172pp (1977)

7. Cowan, J. W.: Genetic studies on somatic recombination in Coprinus lagopus sensu Bull. $\mathrm{Ph}$. D. Thesis, University of London (1964)

8. Giluies, C. B.: An ultrastructural analysis of chromosomal pairing in maize. Compt. Rend. Trav. Lab. Carlsberg 40, 135-161 (1975)

9. Giluies, C. B.: The relationship between synaptonemal complexes, recombination nodules and crossing over in Neurospora crassa bivalents and translocation quadrivalents. Genetics 91, 1-17 (1979)

10. Новоцтн, P.: Chromosome pairing in allohexaploid wheat var. Chinese Spring. Transformation of multivalents into bivalents, a mechanism for exclusive bivalent formation. Carlsberg Res. Commun. 46, 129-173 (1981)

11. Holliday, R.: Recombination and meiosis. Phil. Trans. R. Soc. London B. 277, 359-370 (1977)

12. Holm, P. B.: Three dimensional reconstruction of chromosome pairing during the zygotene stage of meiosis in Lilium longiflorum (Thunb.). Carlsberg Res. Commun. 42, 103-151 (1977)

13. Holm, P. B. \& S. W. Rasmussen: Human meiosis III. Electron microscopical analysis of chromosome pairing in an individual with a balanced translocation 46, XY, t(5p-; 22p +): Carlsberg Res. Commun. 43, 329-350 (1978)

14. Holm, P. B. \& S. W. Rasmussfn: Chromosome pairing, crossing over, chiasma formation and disjunction as revealed by three dimensional reconstructions. In: International Cell Biology 1980-1981, G. Schweiger ed., Springer Verlag. Berlin, Heidelberg \& New York, pp 194-204 (1981)

15. Holm, P. B. \& S. W. Rasmussen: Chromosome pairing, recombination nodules and chiasma formation in diploid Bombyx males. Carlsberg Res. Commun. 45, 483-548 (1981)

16. Lu, B. C.: Fine structure of meiotic chromosomes of the basidiomycete Coprinus lagopus. Exp. Cell Res. 43, 224-227 (1966) 
17. Lu, B. C.: Meiosis in Coprinus lagopus: A comparative study with light and electron microscopy. J. Cell Sci. 2, 529-536 (1967)

18. Lu, B. C.: Genetic recombination in Coprinus. I. Its precise timing as revealed by temperature treatment experiments. Can. J. Genet. Cytol. 11, 834-847 (1969)

19. Lu, B. C.: Meiosis in Coprinus. VIII. A timecourse study of the fusion and division of the spindle pole body during meiosis. J. Cell Biol. 76, 761-766 (1978)

20. Lu, B. C. \& N. B. Raju: Meiosis in Coprinus. II. Chromosome pairing and the lampbrush diplotene stage of meiotic prophase. Chromosoma (Berl.) 29, 305-316 (1970)

21. MOORE, D.: Four new linkage groups in Coprinus lagopus. Genet. Res. Camb. 9, 331342 (1967)

22. Moses, M. J.: Microspreading and the synaptonemal complex in cytogenetic studies. In: Chromosomes Today vol. 6, A. de la Chapelle and M. Sorsa, eds. Elsevier/North Holland Biomedical Press, Amsterdam, New York, Oxford, pp 7182 (1977)

23. Moses, M. J. \& P. A. Poorman: Synaptonemal complex analysis of mouse chromosomal rearrangements. II. Synaptic adjustment in a tandem duplication. Chromosoma (Berl.) 81, 519-535 (1981)

24. Rasmussen, S. W.: The meiotic prophase in Bombyx mori females analyzed by three dimensional reconstructions of synaptonemal complexes. Chromosoma (Berl.) 54, 245-293 (1976)

25. Rasmussen, S. W.: The transformation of the synaptonemal complex into the selimination chromatin in Bombyx mori oocytes. Chromosoma (Berl.) 60, 205-221 (1977)

26. Rasmussen, S. W.: Chromosome pairing in triploid females of Bombyx mori analyzed by three dimensional reconstructions of synaptonemal complexes. Carlsberg Res. Commun. 42, 163197 (1977)

27. Rasmussen, S. W. \& P. B. Holm: Human meiosis II. Chromosome pairing and recombination nodules in human spermatocytes. Carlsberg Res. Commun. 43, 275-327 (1978)

28. Rasmussen, S. W. \& P. B. Holm: Chromosome pairing in autotetraploid Bombyx females. Mechanism for exclusive bivalent formation. Carlsberg Res. Commun. 44, 101-125 (1979)

29. Rasmussen, S. W. \& P. B. Holm: Mechanics of meiosis. Hereditas 93, 187-216 (1980)

30. Rasmussen, S. W., P. B. Holm, B. C. Lu, D. ZICKLER \& J. SAGE: Synaptonemal complex formation and distribution of recombination nodules in pachytene trivalents of triploid Coprinus cinereus. Carlsberg Res. Commun. 46, 347-360 (1981)

31. ZiCKLER, D.: Development of the synaptonemal complex and the srecombination nodules/ dur-ing meiotic prophase in the seven bivalents of the fungus Sordaria macrospora Auersw. Chromosoma (Berl.) 61, 289-316 (1977)

32. ZICKLER, D. \& J. SAGE: Synaptonemal complexes with modified lateral elements in Sordaria humana: Development of and relationship to the srecombination nodules«. Chromosoma (Berl.), in press (1981) 